\title{
Stochastic Path-Integral Analysis of the Continuously Monitored Quantum Harmonic Oscillator
}

\author{
Tathagata Karmakarø, ${ }^{1,2,3,{ }^{*}}$ Philippe Lewalle $\odot,{ }^{1,2,4,5}$ and Andrew N. Jordan ${ }^{3,1,2}$ \\ ${ }^{1}$ Department of Physics and Astronomy, University of Rochester, Rochester, New York 14627, USA \\ ${ }^{2}$ Center for Coherence and Quantum Optics, University of Rochester, Rochester, New York 14627, USA \\ ${ }^{3}$ Institute for Quantum Studies, Chapman University, Orange, California 92866, USA \\ ${ }^{4}$ Department of Chemistry, University of California, Berkeley, California 94720, USA \\ ${ }^{5}$ Berkeley Center for Quantum Information and Computation, Berkeley, California 94720, USA
}

(Received 2 April 2021; accepted 24 January 2022; published 18 February 2022)

\begin{abstract}
We consider the evolution of a quantum simple harmonic oscillator in a general Gaussian state under simultaneous time-continuous weak position and momentum measurements. We deduce the stochastic evolution equations for position and momentum expectation values and the covariance-matrix elements from the characteristic function of the system. By generalizing the Chantasri-Dressel-Jordan (CDJ) formalism (Chantasri et al., 2013, 2015) to this continuous-variable system, we construct its stochastic Hamiltonian and action. Action extremization gives us the equations for the most likely readout paths and quantum trajectories. For steady states of the covariance-matrix elements, the analytical solutions for these most likely paths are obtained. Using the CDJ formalism, we calculate final-state probability densities exactly starting from any initial state. We also demonstrate the agreement between the optimal-path solutions and the averages of simulated clustered stochastic trajectories. Our results provide insights into the time dependence of the mechanical energy of the system during the measurement process, motivating their importance for quantum measurement-engine or refrigerator experiments. Although quantum mechanics fundamentally limits our ability to extract information via simultaneous measurements of noncommuting observables, insights into the dynamics of the system during such a process can lead to a deeper understanding of the physics of measurement.
\end{abstract}

DOI: 10.1103/PRXQuantum.3.010327

\section{INTRODUCTION}

Methods to implement time-continuous measurements of quantum systems [1-4] have emerged in the past three decades; they allow the quantum state to be probed in real time and are indispensable for feedback control [5]. Continuous measurement involves a time sequence of individually weak measurements, in which an observer gently probes the quantum system of interest via its environment and infers the conditional stochastic evolution of the state in real time as he or she receives measurement outcomes. In contrast to a projective measurement, time-continuous weak monitoring, leading to diffusive quantum trajectories, illustrates the gradual "collapse" of the state over time [6]. Our current work draws together two threads from within this area. On the one hand, there has been

\footnotetext{
*tkarmaka@ur.rochester.edu
}

Published by the American Physical Society under the terms of the Creative Commons Attribution 4.0 International license. Further distribution of this work must maintain attribution to the author(s) and the published article's title, journal citation, and DOI. considerable recent experimental progress in the continuous monitoring of quantum harmonic oscillators [7]. On the other, diffusive quantum trajectories have been analyzed via stochastic path integral methods, which have allowed "most likely paths" (MLPs), following an optimal measurement record, to be computed [8-10]. These path integral methods, initially developed by Chantasri, Dressel, and Jordan (CDJ), have been applied to theory and experiment across several examples featuring continuously monitored qubits [8-17]. This formalism has not, however, been applied to the continuously monitored quantum harmonic oscillator or other continuous-variable systems. In this work, we build an action-based description of the quantum trajectories of general Gaussian oscillator states under time-continuous simultaneous position and momentum measurements, using the CDJ formalism. The more widely studied case of continuous position measurement reemerges as a special case. The analysis presented here leads to a complete description of the statistics of the measurement process. Past work on path-integral analysis of the harmonic oscillator coupled to a harmonic environment has extensively investigated the collective (unmonitored) dynamics of the system [18-21]. For continuously 
monitored oscillators, a description based on the stochastic Schrödinger equation has been proposed as well [22].

The harmonic oscillator is ubiquitous across physics, leading to analytically soluble models that are applicable to a wide range of physical systems. It provides a suitable description of optical degrees of freedom and a wide variety of vibrating elements such as cavity mirrors, trapped ions, cantilevers, membranes, and so on. For oscillators, building on the analytical studies of the dynamics of continuous measurement [23-26], quantum state smoothing methods have been explored [27-33]. Hybrid systems, where the quantum state of a mechanical element is probed and read out optically [34,35] have received significant attention too. Experimentally, both position monitoring and control [7,36-43], and measurement-based feedback cooling of resonators [44-49] have been done.

Additionally, mechanical resonators have been used to study the quantum phenomena at macroscopic scales, with the majority of the experiments requiring cooling of the resonator. Recently, experiments have generated entanglement between two mechanical resonators [50,51], between vibrational modes of two diamonds [52], and between optical field-mechanical resonator [53,54]. Complementary to these efforts, superposition states of a membrane resonator [55] and squeezed states of mechanical resonators have been generated successfully [56-60] in optomechanical settings. Furthermore, single-phonon generation and second-order phonon-correlation calculation for nanomechanical resonators on a Hanbury Brown and Twiss setup have been done using optical control [61,62]. As our analysis is able to provide insights into the state dynamics, the statistics of the final state, and the energetics of the measurement processes, it bears direct relevance to recent experimental advances in areas such as optomechanics and superconducting circuits $[7,44,63]$.

This paper is organized as follows. In Sec. II, we describe the stochastic evolution of the system, construct the stochastic action and Hamiltonian, and analytically solve the equations of motions for the optimal paths. In Sec. III, we find the expression for the final-state probability density using the path integral formalism, and in Sec. IV we discuss the energetics of the system. Next, Sec. V explores the connection between stochastic trajectories and optimal paths. Lastly, we present our concluding remarks in Sec. VI.

\section{STOCHASTIC EVOLUTION OF THE SYSTEM}

Our system of interest is a harmonic oscillator that is being time-continuously monitored via two detectors. An example, shown in Fig. 1, is a simple harmonic oscillator and its position and momentum are simultaneously and continuously monitored by an optical measurement setup. (a)

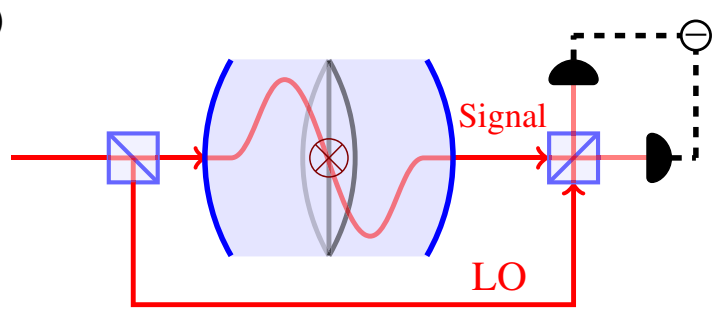

(b)

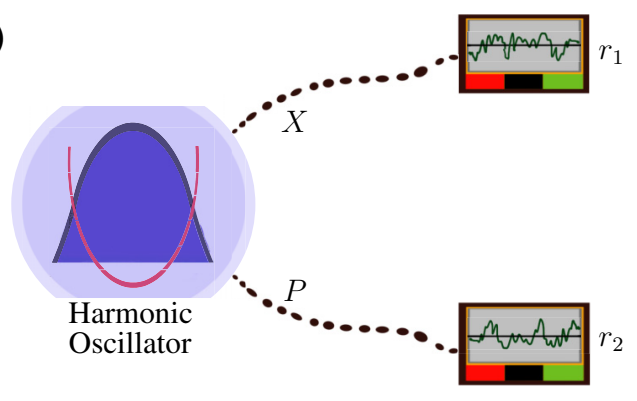

FIG. 1. We sketch an example system of interest, a mechanical oscillator, being probed using an optical field. (a) The case where the position of the oscillator is continuously monitored by performing homodyne detection on the transmitted light. (b) The schematic of the setup for simultaneous position and momentum measurements. In this case, the harmonic oscillator under consideration is coupled to two measurement devices that independently read out the position and momentum of the oscillator simultaneously and continuously. A similar measurement scheme has been implemented successfully for qubits [64].

\section{A. State description and evolution}

The closed quantum harmonic oscillator exhibits unitary evolution due to its Hamiltonian.

$$
\hat{H}=\frac{1}{2 m} \hat{p}^{2}+\frac{1}{2} m \omega^{2} \hat{x}^{2} .
$$

We define the dimensionless position and momentum observables as $\hat{X}=\sqrt{m \omega / \hbar} \hat{x}$ and $\hat{P}=\frac{\hat{p}}{\sqrt{\hbar m \omega}}$. We also define $\tau=\omega t$ and $\hat{H}^{\prime}=\hat{H} / \hbar \omega$. From now on, we refer to these four dimensionless quantities as the position, momentum, time, and Hamiltonian (or energy), respectively.

For experiments of the type shown in Fig. 1, we consider weak continuous measurements of a Gaussian type. Such measurements are realized by interacting two auxiliary systems, both assumed to be continuous-variable systems themselves, with the system with interaction Hamiltonian $\hat{H}_{I}^{\prime} \delta \tau=\sqrt{\frac{\delta \tau}{2}}\left(\hat{P}_{1} \hat{X}+\hat{P}_{2} \hat{P}\right)$, where $\hat{P}_{1}$ and $\hat{P}_{2}$ are dimensionless momenta of the auxiliary systems [65]. Here, $\delta \tau$ is the duration of the measurement. Also, both auxiliary systems are initially in Gaussian states with dimensionless position variances $\tau_{1} / 2$ and $\tau_{2} / 2$, respectively. Detector readouts $r_{1}$ and $r_{2}$ correspond to the projection of these 
two systems into position eigenstates $x_{1}=\sqrt{\frac{\delta \tau}{2}} r_{1}$ and $x_{2}=$ $\sqrt{\frac{\delta \tau}{2}} r_{2}$, respectively [12,17] (as is consistent with, e.g., homodyne detection of each auxiliary system). Such a measurement scheme realizes a positive operator-valued measure (POVM). The Kraus operator can be found using

$$
\sqrt{d r_{1} d r_{2}} \hat{M}\left(r_{1}, r_{2}\right)=\sqrt{d r_{1} d r_{2}}\left\langle x_{1}, x_{2}\left|\exp \left\{-i \hat{H}_{I}^{\prime} \delta \tau\right\}\right| i\right\rangle .
$$

Here, $|i\rangle$ denotes the initial Gaussian state of the two auxiliary systems. As we only consider weak measurements (long collapse time scales), i.e., $\delta \tau \ll \tau_{1}, \tau_{2}$, up to $O(\delta \tau) \hat{M}\left(r_{1}, r_{2}\right)$ can be written as a product of two Kraus operators $\hat{M}_{X}\left(r_{1}\right)$ and $\hat{M}_{P}\left(r_{2}\right)$, corresponding to position and momentum measurements. These Kraus operators [66] representing variable-strength position or momentum measurements are given, respectively, by

$$
\hat{M}_{X}\left(r_{1}\right)=\left(\frac{\delta \tau}{2 \pi \tau_{1}}\right)^{\frac{1}{4}} \exp \left[-\frac{\delta \tau}{4 \tau_{1}}\left(r_{1} \mathbb{1}-\hat{X}\right)^{2}\right]
$$

and

$$
\hat{M}_{P}\left(r_{2}\right)=\left(\frac{\delta \tau}{2 \pi \tau_{2}}\right)^{\frac{1}{4}} \exp \left[-\frac{\delta \tau}{4 \tau_{2}}\left(r_{2} \mathbb{1}-\hat{P}\right)^{2}\right],
$$

with $r_{1}$ and $r_{2}$ as the readouts of the two measurements. We can see that $\int_{-\infty}^{\infty} d r_{1} \hat{M}_{X}\left(r_{1}\right)^{\dagger} \hat{M}_{X}\left(r_{1}\right)=\mathbb{1}$ and $\int_{-\infty}^{\infty} d r_{2} \hat{M}_{P}\left(r_{2}\right)^{\dagger} \hat{M}_{P}\left(r_{2}\right)=\mathbb{1}$, indicating that these operators form elements of a POVM and represent a form of complete information collection from the environmental degrees of freedom interacting with the system.

For such measurements made over a time interval from $\tau$ to $\tau+\delta \tau$, the state (density matrix $\hat{\rho}$ ) can then be updated via [1-4]

$$
\begin{aligned}
& \hat{\rho}(\tau+\delta \tau) \\
& \quad=\frac{e^{-i \hat{H}^{\prime} \delta \tau} \hat{M}_{X}\left(r_{1}\right) \hat{M}_{P}\left(r_{2}\right) \hat{\rho}(\tau) \hat{M}_{P}^{\dagger}\left(r_{2}\right) \hat{M}_{X}^{\dagger}\left(r_{1}\right) e^{i \hat{H}^{\prime} \delta \tau}}{\operatorname{Tr}\left[\hat{M}_{X}\left(r_{1}\right) \hat{M}_{P}\left(r_{2}\right) \hat{\rho}(\tau) \hat{M}_{P}^{\dagger}\left(r_{2}\right) \hat{M}_{X}^{\dagger}\left(r_{1}\right)\right]},
\end{aligned}
$$

which includes the update conditioned on the outcomes of simultaneous weak $\hat{X}$ and $\hat{P}$ measurements, as well as the unitary evolution due to the bare Hamiltonian Eq. (1). The choice of order of operations above is arbitrary, as the commutators between the different operators only play a role to $O\left(\delta \tau^{2}\right)$ and can be neglected in the limit of weak continuous measurement. Although the Heisenberg uncertainty principle forbids joint projective measurement of complimentary observables, simultaneous weak (nonprojective) measurement can be done as described by Jordan and Büttiker for qubits [67]. Notably, the protocol by
Arthurs and Kelly for harmonic oscillators provides a way to achieve the minimum uncertainty limit for such simultaneous measurements $[68,69]$. Physical implementations of this process on optical degrees of freedom are typically achieved via heterodyne detection [70] or quantum-limited phase-preserving amplification [71] (either of which can be associated with projectors in the coherent state basis). Further ideas about continuous monitoring of noncommuting observables have been developed in the literature concerning qubit measurements as well (see, e.g., Refs. $[14,16,64,72-75])$.

Our analysis is restricted to general Gaussian states (see Appendix A) of the oscillator. The common states, including coherent states, and the measurements are both Gaussian, making such restriction a reasonable choice $[23,24,76,77]$. We see below that both unitary and measurement dynamics of the type given in Eq. (4) preserve the Gaussian form of the states. The evolution of such states can be expressed in terms of the expectation values of position and momentum, their variances, and their covariance. The characteristic function at time $\tau, \chi_{\tau}(\xi)$ (see Appendix A) is by definition a Gaussian in $\boldsymbol{\xi}=\left(\xi_{1}, \xi_{2}\right)^{\top}$. At time $\tau+\delta \tau$, the characteristic function is [from Eq. (A1)]

$$
\chi_{\tau+\delta \tau}(\boldsymbol{\xi})=\operatorname{Tr}\left[\hat{\rho}(\tau+\delta \tau) e^{i \boldsymbol{\xi}^{\top} \cdot \hat{\mathcal{R}}}\right]
$$

with $\hat{\mathcal{R}}=(\hat{X}, \hat{P})^{\top}$. It can be shown that to $O(\delta \tau)$, we have

$$
\chi_{\tau+\delta \tau}(\boldsymbol{\xi})=\chi_{\tau}(\boldsymbol{\xi}) e^{\kappa \delta \tau},
$$

with $\kappa$ given as

$$
\begin{aligned}
& \frac{1}{2}\left(q_{3} \xi_{1}+q_{4} \xi_{2}\right) \xi_{2}-\frac{1}{2}\left(q_{4} \xi_{1}+q_{5} \xi_{2}\right) \xi_{1}-\frac{1}{8 \tau_{2}} \xi_{1}^{2}-\frac{1}{8 \tau_{1}} \xi_{2}^{2} \\
& \quad+\frac{1}{8 \tau_{1}}\left(q_{3} \xi_{1}+q_{4} \xi_{2}\right)^{2}+\frac{1}{8 \tau_{2}}\left(q_{4} \xi_{1}+q_{5} \xi_{2}\right)^{2} \\
& \quad-i q_{1} \xi_{2}+i \xi_{1} q_{2}+\frac{i}{8 \tau_{1}}\left(q_{3} \xi_{1}+q_{4} \xi_{2}\right)\left(r_{1}-q_{1}\right) \\
& \quad+\frac{i}{8 \tau_{2}}\left(q_{4} \xi_{1}+q_{5} \xi_{2}\right)\left(r_{2}-q_{2}\right) .
\end{aligned}
$$

The variables $q_{1}, \ldots, q_{5}$ are defined as the scaled cumulants

$$
\begin{aligned}
& q_{1}=\langle\hat{X}\rangle, q_{2}=\langle\hat{P}\rangle, q_{4}=\langle\hat{X} \hat{P}+\hat{P} \hat{X}\rangle-2\langle\hat{X}\rangle\langle\hat{P}\rangle, \\
& q_{3}=2\left(\left\langle\hat{X}^{2}\right\rangle-\langle\hat{X}\rangle^{2}\right), q_{5}=2\left(\left\langle\hat{P}^{2}\right\rangle-\langle\hat{P}\rangle^{2}\right) .
\end{aligned}
$$

The form of Eq. (6b) confirms the preservation of Gaussianity of the state $[24,29,76]$. Equivalently, we can characterize the state evolution in terms of the variables defined in Eq. (7). From Eqs. (A2), (6a), and (6b), the stochastic equations in $q_{1}$ and $q_{2}$ can be written in the Langevin form: 


$$
\dot{\mathbf{q}}=\mathbf{\Omega} \cdot \mathbf{q}+\mathbf{b} \cdot \frac{d \mathbf{W}}{d \tau}=\mathbf{\Omega} \cdot \mathbf{q}+\mathbf{b} \cdot(\mathbf{R}-\mathbf{X})=\mathcal{F}(\mathbf{q}, \mathbf{r}),
$$

where $\mathbf{q}=\left(q_{1}, q_{2}\right)^{\top} ; \boldsymbol{\Omega}$ and $\mathbf{b}$ are the matrices

$$
\boldsymbol{\Omega}=\left(\begin{array}{cc}
0 & 1 \\
-1 & 0
\end{array}\right), \mathbf{b}=\left(\begin{array}{cc}
\frac{q_{3}}{2 \sqrt{\tau_{1}}} & \frac{q_{4}}{2 \sqrt{\tau_{2}}} \\
\frac{q_{4}}{2 \sqrt{\tau_{1}}} & \frac{q_{5}}{2 \sqrt{\tau_{2}}}
\end{array}\right)
$$

$d \mathbf{W}$ is the vector of Wiener noises $d W_{1}$ and $d W_{2}$, which are related to the measurement readouts by

$$
r_{1}=\langle\hat{X}\rangle+\sqrt{\tau_{1}} \frac{d W_{1}}{d \tau}, r_{2}=\langle\hat{P}\rangle+\sqrt{\tau_{2}} \frac{d W_{2}}{d \tau},
$$

and which may each be interpreted as a sum of signal and noise terms. Here, $r_{1}$ measures the position of the oscillator, while $r_{2}$ measures its momentum. For notational convenience, we define $\mathbf{R}=\mathbf{X}+d \mathbf{W} / d t$ with

$$
\mathbf{R}=\left(\begin{array}{c}
r_{1} / \sqrt{\tau_{1}} \\
r_{2} / \sqrt{\tau_{2}}
\end{array}\right), \quad \mathbf{X}=\left(\begin{array}{c}
\langle\hat{X}\rangle / \sqrt{\tau_{1}} \\
\langle\hat{P}\rangle / \sqrt{\tau_{2}}
\end{array}\right) .
$$

The diffusion tensor $\mathbf{b}$ depends on $q_{3}, q_{4}$, and $q_{5}$. The covariance-matrix elements evolve according to the deterministic equations

$$
\begin{aligned}
& \dot{q}_{3}=2 q_{4}-\frac{1}{2 \tau_{1}} q_{3}^{2}-\frac{1}{2 \tau_{2}} q_{4}^{2}+\frac{1}{2 \tau_{2}}, \\
& \dot{q}_{4}=q_{5}-q_{3}-\frac{1}{2 \tau_{1}} q_{3} q_{4}-\frac{1}{2 \tau_{2}} q_{4} q_{5}, \\
& \dot{q}_{5}=-2 q_{4}-\frac{1}{2 \tau_{1}} q_{4}^{2}-\frac{1}{2 \tau_{2}} q_{5}^{2}+\frac{1}{2 \tau_{1}},
\end{aligned}
$$

consistent with Refs. [23,24].

These equations of motion are equivalent to those obtained from a stochastic master equation, which can be derived from Eq. (4), the Itô form of which reads [23]

$$
\begin{aligned}
d \rho= & -i\left[\hat{H}^{\prime}, \hat{\rho}\right] d \tau+\frac{1}{4 \tau_{1}}\left[\hat{X} \hat{\rho} \hat{X}-\frac{1}{2}\left(\hat{X}^{2} \hat{\rho}+\hat{\rho} \hat{X}^{2}\right)\right] d \tau \\
& +\frac{1}{4 \tau_{2}}\left[\hat{P} \hat{\rho} \hat{P}-\frac{1}{2}\left(\hat{P}^{2} \hat{\rho}+\hat{\rho} \hat{P}^{2}\right)\right] d \tau \\
& +\frac{1}{\sqrt{4 \tau_{1}}}[\hat{X}-\langle\hat{X}\rangle, \hat{\rho}]_{+} d W_{1} \\
& +\frac{1}{\sqrt{4 \tau_{2}}}[\hat{P}-\langle\hat{P}\rangle, \hat{\rho}]_{+} d W_{2}
\end{aligned}
$$

Here, $[\cdots, \ldots]_{+}$denotes the anticommutator of two observables. It is noteworthy that no distinction needs to be made between the Itô and Stratonovich forms of Eqs. (8) and (11) for these Gaussian-state equations because of the linear nature of the stochastic terms.
Unlike the quadrature variables $q_{1}$ and $q_{2}$, the covariance-matrix elements $q_{3}, q_{4}$, and $q_{5}$, evolve deterministically, as they depend neither directly nor indirectly on the measurement record(s). Therefore, their evolution is governed by ordinary, rather than stochastic, differential equations. The typical behavior of these covariance-matrix elements is shown in Fig. 2. We can see that for large $\tau$, all of them tend to the fixed-point values of Eq. (11) (proof of this statement is given in Appendix B). For arbitrary measurement strength, the fixed points to which they settle are

$$
\begin{aligned}
& \tilde{q}_{4}=\frac{1}{2 \gamma_{2}}\left(\sqrt{\frac{\gamma_{1}^{2}}{\tau_{2}^{2}}+4 \gamma_{2}^{2}}-\frac{\gamma_{1}}{\tau_{2}}\right), \\
& \tilde{q}_{3}=\sqrt{\frac{4 \tau_{1} \tilde{q}_{4}}{\gamma_{2}}\left(1+\frac{1}{4 \tau_{2}^{2}}\right)}, \\
& \tilde{q}_{5}=\sqrt{\left(1+4 \tau_{1}^{2}\right) \frac{\tilde{q}_{4}}{\gamma_{2} \tau_{1}}} .
\end{aligned}
$$

We use the definitions $\gamma_{1}=1+4 \tau_{1} \tau_{2}$ and $\gamma_{2}=1-\tau_{1} / \tau_{2}$. For $\tau_{1}=\tau_{2}$ (equal measurement strength), the fixed points are

$$
\tilde{q}_{3}=1, \tilde{q}_{4}=0, \tilde{q}_{5}=1 .
$$

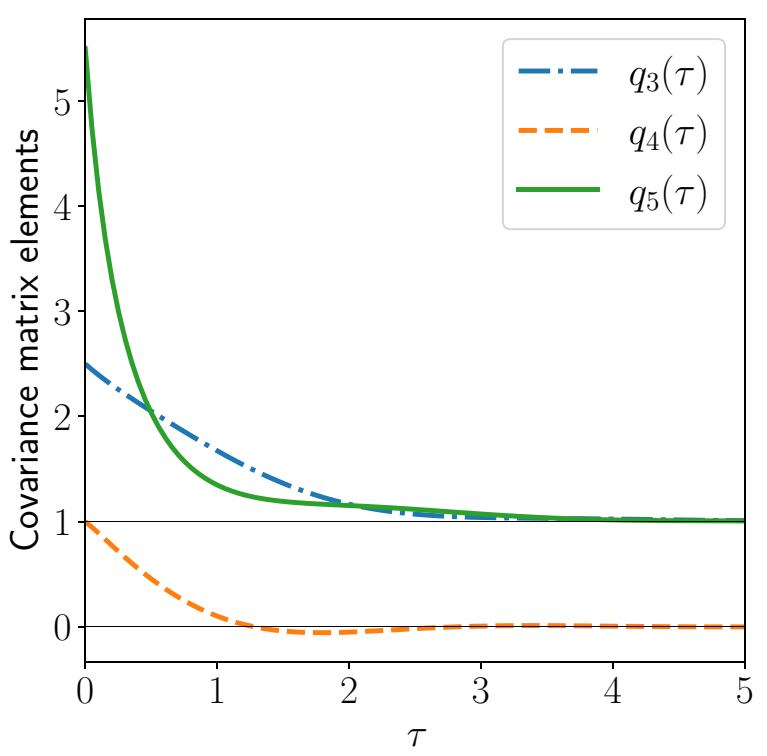

FIG. 2. The time evolution of the covariance-matrix elements for equal measurement strength $\left(\tau_{1}=\tau_{2}=1\right)$. The blue dashdotted line shows $q_{3}$, the orange dashed line shows $q_{4}$, and the green solid line corresponds to $q_{5}$ defined in Eq. (7). The initial values are $q_{3}(0)=2.5, q_{4}(0)=1$, and $q_{5}(0)=5.5$. The covariance-matrix elements tend to the fixed points given in Eq. (13b) for $\tau \rightarrow \infty$. As the determinant goes to 1 , this evolution represents purification of the state by continuous measurement. 


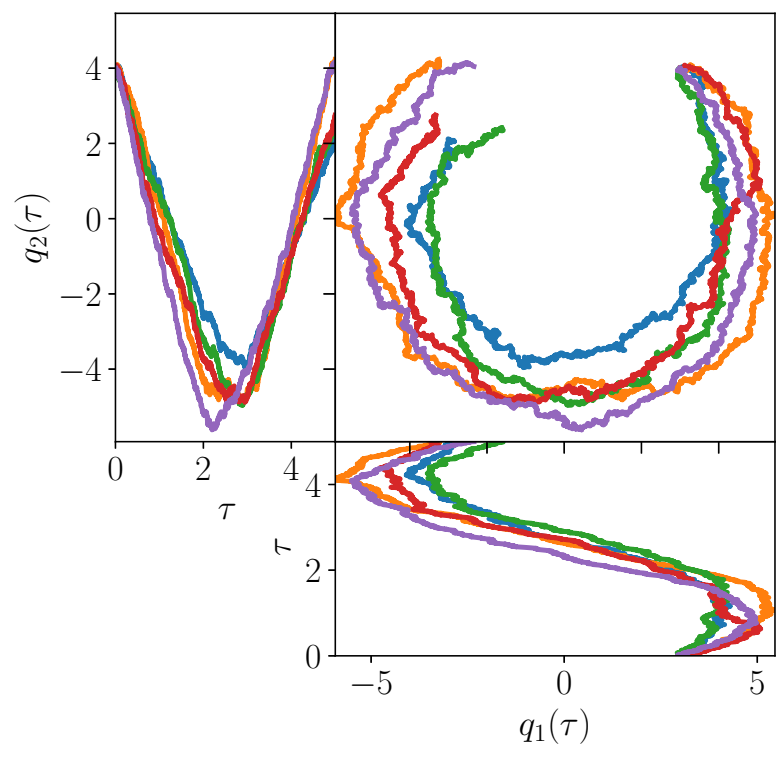

FIG. 3. Sample stochastic trajectories until final time $\tau_{f}=5.0$ for $\tau_{1}=\tau_{2}=1$, given initial conditions $q_{1}(0)=3$ and $q_{2}(0)=$ 4. The top-left panel shows $q_{2}$ versus time, the bottom-right panel shows time versus $q_{1}$, and the top-right panel shows $q_{2}$ versus $q_{1}$. The spiral patterns in the trajectories arise from diffusion about the oscillatory unitary dynamics.

The oscillator as a whole tends to a pure state as $q_{3} q_{5}-$ $q_{4}^{2} \rightarrow 1$ (see Appendices A and B). For the case of equal measurement strength emphasized in this work, the covariance matrix converges to the identity matrix and we obtain a coherent state at $\tau \rightarrow \infty$ [77]. In what follows, we take the covariance-matrix elements to have these fixed values for simplicity and ignore the time evolution of $q_{3}, q_{4}$, and $q_{5}$, that is, we start our time after the covariance matrix reaches its steady state.

We now begin to investigate the stochastic evolution of the quadratures. We use the Euler method to integrate Eq. (8). The global truncation error is proportional to $\delta \tau$. Figure 3 shows sample stochastic trajectories, each generated from the same initial condition, where we see diffusion about the phase-space ellipses given by the unitary evolution.

\section{B. Stochastic action and Hamiltonian}

We briefly recap the CDJ stochastic path integral (SPI) formalism [8-10] and adapt it to the case of interest. The state at time $\tau_{k}$ is given by $\rho_{k}$ (which in turn is completely characterized by $\mathbf{q}_{k}$ ), and the initial and final $\mathbf{q}$ are given by $\mathbf{q}_{i}$ and $\mathbf{q}_{f}$. The joint probability density of having the readouts $\left\{r_{1 k}, r_{2 k}\right\}$ and states $\mathbf{q}_{k}$ is given by

$$
\mathcal{P}=\delta^{2}\left(\mathbf{q}_{0}-\mathbf{q}_{i}\right) \delta^{2}\left(\mathbf{q}_{n}-\mathbf{q}_{f}\right) \prod_{k=0}^{n-1} P\left(\mathbf{q}_{k+1}, \mathbf{r}_{k} \mid \mathbf{q}_{k}\right) .
$$

We can write the conditional probability on the right-hand side of Eq. (14) as $P\left(\mathbf{q}_{k+1}, \mathbf{r}_{k} \mid \mathbf{q}_{k}\right)=P\left(\mathbf{q}_{k+1} \mid \mathbf{q}_{k}, \mathbf{r}_{k}\right) P\left(\mathbf{r}_{k} \mid \mathbf{q}_{k}\right)$. For the purposes of writing the stochastic path integral, we only need to focus on the variables that are in fact stochastic. The stochasticity of the equations is entirely contained in the readouts drawn from the probability density

$$
\begin{aligned}
P\left(\mathbf{r}_{k} \mid \mathbf{q}_{k}\right) & =P\left(\mathbf{r}_{k} \mid \rho_{k}\right) \\
& =\operatorname{Tr}\left[\hat{M}_{X}\left(r_{1 k}\right) \hat{M}_{P}\left(r_{2 k}\right) \hat{\rho}\left(\tau_{k}\right) \hat{M}_{P}^{\dagger}\left(r_{1 k}\right) \hat{M}_{X}^{\dagger}\left(r_{2 k}\right)\right],
\end{aligned}
$$

and the coordinates $q_{1}$ and $q_{2}$ that evolve as a function of those readouts. Those quadrature coordinates evolve deterministically when we condition on the values of the stochastic readouts $r_{1}$ and $r_{2}$, and are thereby described by $P\left(\mathbf{q}_{k+1} \mid \mathbf{q}_{k}, \mathbf{r}_{k}\right)=\delta^{2}\left[\mathbf{q}_{k+1}-\mathbf{q}_{k}-\right.$ $\left.\delta \tau \mathcal{F}\left(\mathbf{q}_{k}, \mathbf{r}_{k}\right)\right]$. The remaining three coordinates (describing the covariance matrix) evolve deterministically (independently of $r_{1}, r_{2}, q_{1}$, and $q_{2}$ ) and can therefore largely be ignored in constructing the path integral; they appear as time-dependent driving terms if allowed to evolve or merely as constants if they are at their steady state.

Each of the delta functions associated with $P\left(\mathbf{q}_{k+1} \mid \mathbf{q}_{k}\right.$, $\mathbf{r}_{k}$ ), as well as the boundary conditions in Eq. (14), can be expressed as a Fourier integral of the form $\delta(q)=$ $\frac{1}{2 \pi i} \int_{-i \infty}^{i \infty} \exp \{-p q\} d p$. With this in mind, we can write $P\left(\mathbf{r}_{k} \mid \mathbf{q}_{k}\right) \approx \exp \left[C+\delta \tau \mathcal{G}\left(\mathbf{q}_{k}, \mathbf{r}_{k}\right)+O\left(\delta \tau^{2}\right)\right]$ where $C=$ $\log \left(\delta \tau / 2 \pi \sqrt{\tau_{1} \tau_{2}}\right)$ and

$$
\mathcal{G}(\mathbf{q}, \mathbf{r})=-\frac{\left(r_{1}-q_{1}\right)^{2}}{2 \tau_{1}}-\frac{\left(r_{2}-q_{2}\right)^{2}}{2 \tau_{2}}-\frac{q_{3}}{4 \tau_{1}}-\frac{q_{5}}{4 \tau_{2}},
$$

where the first two terms indicate that the noise on each measurement is Gaussian (i.e., noise about the means $q_{1}$ or $q_{2}$ is characterized by a variance $\tau_{1} / \delta \tau$ or $\tau_{2} / \delta \tau$, respectively), consistent with Eq. (10). The last two terms $\mathrm{g}_{1} \equiv$ $q_{3} / 4 \tau_{1}=\operatorname{var}(\hat{X}) / 2 \tau_{1}$ and $g_{2} \equiv q_{5} / 4 \tau_{2}=\operatorname{var}(\hat{P}) / 2 \tau_{2}$ can be interpreted as a measure of the local rate of information gain expected from each measurement [3,78-80]. Using Fourier forms of the $\delta$ functions (thereby introducing a pair of variables $p_{1}$ and $p_{2}$ conjugate to $q_{1}$ and $q_{2}$ ) we may now express the right-hand side of Eq. (14) as a stochastic path integral; taking a time-continuum limit $[8,9]$ leads to

$$
\begin{aligned}
\mathcal{P}= & \int \mathcal{D} \mathbf{p} e^{\mathcal{S}}=\int \mathcal{D} \mathbf{p} \\
& \exp \left[\int_{0}^{\tau_{f}} d \tau[-\mathbf{p} \cdot \dot{\mathbf{q}}+\mathcal{H}(\mathbf{p}, \mathbf{q}, \mathbf{r})]\right],
\end{aligned}
$$

where a stochastic action $\mathcal{S}$ is defined in terms of a stochastic Hamiltonian

$$
\begin{aligned}
\mathcal{H} & =\mathbf{p}^{\top} \cdot \mathcal{F}(\mathbf{q}, \mathbf{r})+\mathcal{G}(\mathbf{q}, \mathbf{r}) \\
& =\mathbf{p}^{\top} \cdot(\boldsymbol{\Omega} \cdot \mathbf{q}+\mathbf{b} \cdot[\mathbf{R}-\mathbf{X}])-\frac{1}{2}(\mathbf{R}-\mathbf{X})^{2}-\mathbf{g}
\end{aligned}
$$


Here, $\mathbf{p}=\left(p_{1}, p_{2}\right)^{\top}$. We use the shorthand $\mathbf{g}=\mathrm{g}_{1}+\mathrm{g}_{2}=$ $q_{3} / 4 \tau_{1}+q_{5} / 4 \tau_{2}$. We stress that $\mathbf{p}$ and $\mathcal{H}$ are unrelated to the mechanical momentum and Hamiltonian.

\section{Equation of motion for optimal path}

The CDJ formalism guides us to the action $\mathcal{S}$. Given initial and final states, the variational solutions $\delta \mathcal{S}=0$ are trajectories following extremal-probability measurement records that connect the given boundary conditions given in Eq. (14). The equations for these trajectories can be summarized as the three equations $\partial_{\mathbf{R}} \mathcal{H}=0, \dot{\mathbf{q}}=\partial_{\mathbf{p}} \mathcal{H}$, and $\dot{\mathbf{p}}=-\partial_{\mathbf{q}} \mathcal{H}$ analogous to Hamiltonian's equations of motion. We refer to the corresponding solutions as optimal paths (OPs), or as MLPs when they maximize the record probability $[8,14]$.

The readouts corresponding to the optimal paths (also called optimal readouts) can be calculated from $\partial_{\mathbf{R}} \mathcal{H}=0$, giving

$$
\mathbf{R}^{\star}=\mathbf{X}+\mathbf{b}^{\top} \cdot \mathbf{p}
$$

where $\mathbf{X}$ is the signal and $\mathbf{b}^{\top} \cdot \mathbf{p}$ is the optimal noise.

In the event that the covariance-matrix elements $q_{3}$, $q_{4}$, and $q_{5}$ are initialized at their steady-state values [see Eqs. (13a) and (13b)], the "stochastic energy" $E_{s}=\mathcal{H}$ is conserved in OP evolution and we are able to find analytical solutions to the OP equations of motion.

Using the above readout values in Eq. (18) (or, equivalently, integrating them out) and fixing the covariancematrix elements to their steady state gives another form of Eq. (18):

$$
\mathcal{H}^{\star}(\mathbf{p}, \mathbf{q})=\frac{1}{2} \mathbf{p}^{\top} \cdot \mathbf{B} \cdot \mathbf{p}+\mathbf{p}^{\top} \cdot \boldsymbol{\Omega} \cdot \mathbf{q}-\mathbf{g} .
$$

Here, we use the shorthand $\mathbf{B} \equiv \mathbf{b} \mathbf{b}^{\top}$. The matrix $\mathbf{B}$ depends on the covariance-matrix elements and is a square positive symmetric matrix by construction. The equations of motion for the optimal paths can be found using Hamilton's equations $\dot{\mathbf{q}}=\partial_{\mathbf{p}} \mathcal{H}^{\star}$ and $\dot{\mathbf{p}}=-\partial_{\mathbf{q}} \mathcal{H}^{\star}$. From Eq. (20), these can be written as

$$
\begin{aligned}
& \dot{\mathbf{q}}=\mathbf{B} \cdot \mathbf{p}+\mathbf{\Omega} \cdot \mathbf{q}, \\
& \dot{\mathbf{p}}=\mathbf{\Omega} \cdot \mathbf{p},
\end{aligned}
$$

where we recall the matrices $\mathbf{b}$ and $\boldsymbol{\Omega}$ are defined in (9). The equations for $p_{1}$ and $p_{2}$ give rise to sinusoidal solutions. Therefore, the quadrature evolution can be viewed as a forced harmonic oscillator on resonance. When $\mathbf{B}$ is constant [i.e., the covariance-matrix elements are assumed to be time independent with values from Eq. (13a) or Eq. (13b)], we expect to have oscillatory solutions for the quadratures as well, with the coefficients of sine and cosine exhibiting linear time dependence. If we relax the assumption about the covariance-matrix elements, however, $q_{3}, q_{4}$, and $q_{5}$ act as some known but timedependent driving term in the stochastic Hamiltonian. In that case, the stochastic energy is no longer conserved and the OP equations of motion may be solved numerically.

\section{Solution for optimal path}

We now discuss the analytical solution of Eq. (21) when $\tau_{1}=\tau_{2}=\mathcal{T}$ (i.e., in the case of equal measurement strength) for simplicity, with the solution of the general case given in the Appendix D. The covariance-matrix elements take the values given in Eq. (13b) and the matrix B can be evaluated to be $(1 / 4 \mathcal{T}) \mathbb{1}_{2}$, i.e., proportional to the $2 \times 2$ identity matrix.

The analytical solution of Eq. (21) traversing from initial conditions $q_{1 i}$ and $q_{2 i}$ to the final conditions $q_{1 f}$ and $q_{2 f}$, over the time interval $\tau_{f}$, is

$$
\begin{aligned}
q_{1}(\tau)= & \frac{\tau}{\tau_{f}}\left[q_{1 f} \cos \left(\tau_{f}-\tau\right)-q_{2 f} \sin \left(\tau_{f}-\tau\right)\right] \\
& +\left(1-\frac{\tau}{\tau_{f}}\right)\left(q_{1 i} \cos \tau+q_{2 i} \sin \tau\right), \\
q_{2}(\tau)= & \frac{\tau}{\tau_{f}}\left[q_{1 f} \sin \left(\tau_{f}-\tau\right)+q_{2 f} \cos \left(\tau_{f}-\tau\right)\right] \\
& +\left(1-\frac{\tau}{\tau_{f}}\right)\left(-q_{1 i} \sin \tau+q_{2 i} \cos \tau\right), \\
p_{1}(\tau)= & \frac{4 \mathcal{T}}{\tau}\left[q_{1 f} \cos \left(\tau_{f}-\tau\right)-q_{2 f} \sin \left(\tau_{f}-\tau\right)\right. \\
& \left.-\left(q_{1 i} \cos \tau+q_{2 i} \sin \tau\right)\right], \\
p_{2}(\tau)= & \frac{4 \mathcal{T}}{\tau_{f}}\left[q_{1 f} \sin \left(\tau_{f}-\tau\right)+q_{2 f} \cos \left(\tau_{f}-\tau\right)\right. \\
& \left.-\left(-q_{1 i} \sin \tau+q_{2 i} \cos \tau\right)\right] .
\end{aligned}
$$

As discussed previously, the solutions for $p_{1}$ and $p_{2}$ are sinusoidal, while $q_{1}$ and $q_{2}$ are sinusoidal with coefficients growing linearly with $\tau$. Note that there is no dependence on strength $\mathcal{T}$ of measurement in $q_{1}(\tau)$ and $q_{2}(\tau)$. The strength only appears in the probability of the most likely path. We see that the boundary conditions of the position and momentum define a unique solution. As a result, "multipath solutions" [14-16,81] do not exist in this system and we may further understand that every OP solution is in fact a most likely path, simplifying the interpretation of the solutions considerably.

The action is often treated as a generating function that transforms between different dynamical variables at different times [82-84]. The relation $\mathbf{p}_{f}=\partial_{\mathbf{q}_{f}} \mathcal{S}$ is of particular interest in our present case, as it indicates that the globally most likely OP is the one that terminates at $\mathbf{p}_{f}=0$ [14]. Its final coordinates,

$$
\begin{aligned}
& \mathcal{Q}_{1 f}=q_{1 i} \cos \tau_{f}+q_{2 i} \sin \tau_{f}, \\
& \mathcal{Q}_{2 f}=-q_{1 i} \sin \tau_{f}+q_{2 i} \cos \tau_{f},
\end{aligned}
$$


denote the globally most likely final state under the measurement dynamics, given that the initial state $\mathbf{q}_{i}$ at $\tau=0$. This particular MLP is a circular path in the phase space following the unitary dynamics alone and, in fact, has $\mathbf{p}=0$ over its entire evolution in our present example. In our system, the globally most likely path is also the average path over all possible stochastic trajectories. Other choices of $p_{1}$ and $p_{2}$ correspond to postselection on other possible (but less likely) final states allowed under the diffusive measurement dynamics.

\section{FINAL-STATE PROBABILITY DENSITY}

In this section, we exactly calculate $P\left(\mathbf{q}_{f} \mid \mathbf{q}_{i}\right)$, i.e., the probability density of the final state $\mathbf{q}_{f}\left(\tau_{f}\right)$, starting from the initial state $\mathbf{q}_{i}$. We can calculate this quantity by integrating the probability density given in Eq. (17) over all possible quadratures and readouts:

$$
\begin{aligned}
P\left(\mathbf{q}_{f} \mid \mathbf{q}_{i}\right)= & \int \mathcal{D} \mathbf{p} \mathcal{D} \mathbf{q} \mathcal{D} \mathbf{r} e^{\mathcal{S}}=\int \mathcal{D} \mathbf{p} \mathcal{D} \mathbf{q} \mathcal{D} \mathbf{r} \\
& \times \exp \left\{\int_{0}^{\tau_{f}} d \tau[-\mathbf{p} \cdot \dot{\mathbf{q}}+\mathcal{H}(\mathbf{p}, \mathbf{q}, \mathbf{r})]\right\} .
\end{aligned}
$$

Using Eq. (18) and integrating with respect to the readouts (see Appendix E for details), we obtain

$$
P\left(\mathbf{q}_{f} \mid \mathbf{q}_{i}\right)=\mathcal{N}_{1} \int \mathcal{D} \mathbf{p} \mathcal{D} \mathbf{q} e^{-\int_{0}^{\tau_{f}} d \tau\left(\mathbf{p} \cdot \dot{\mathbf{q}}-\frac{1}{2} \mathbf{p}^{\top} \cdot \mathbf{B} \cdot \mathbf{p}-\mathbf{p}^{\top} \cdot \mathbf{\Omega} \cdot \mathbf{q}+\mathrm{g}\right)},
$$

with $\mathcal{N}_{1}$ as a constant that is only dependent on $\tau_{f}$ and the measurement strengths. This recovers the stochastic Hamiltonian (20). The functional integration over $\mathbf{p}$ and q can be carried out to find

$$
P\left(\mathbf{q}_{f} \mid \mathbf{q}_{i}\right)=\mathcal{N} \exp \left(-\frac{1}{2} \int_{0}^{\tau_{f}} d \tau \mathbf{p}_{\mathrm{OP}}^{\top} \cdot \mathbf{B} \cdot \mathbf{p}_{\mathrm{OP}}\right),
$$

where $\mathcal{N}$ is the normalization constant and pop denotes the analytical solution for the optimal-path momenta with given boundary conditions. For the equal measurement strengths, this reduces to

$$
P\left(\mathbf{q}_{f} \mid \mathbf{q}_{i}\right)=\frac{2 \mathcal{T}}{\pi \tau_{f}} \exp \left[-\frac{2 \mathcal{T}}{\tau_{f}}\left(\Delta Q_{1}^{2}+\Delta Q_{2}^{2}\right)\right]
$$

where $\Delta Q_{1}=q_{1 f}-\mathcal{Q}_{1 f}$ and $\Delta Q_{2}=q_{2 f}-\mathcal{Q}_{2 f}$. We see that the solution depends on the measurement time $\mathcal{T}$ relative to the duration $\tau_{f}$ and is Gaussian in $\Delta Q_{1}$ and $\Delta Q_{2}$. The variance is $\tau_{f} / \mathcal{T}$. This is consistent with the fact that stronger measurement (smaller values of $\mathcal{T}$ ) leads to larger diffusion in the trajectories due to measurement back action. Figure 4 compares these analytical results from path integral calculations with 100000 trajectories generated from Eq. (8), confirming the above calculation.

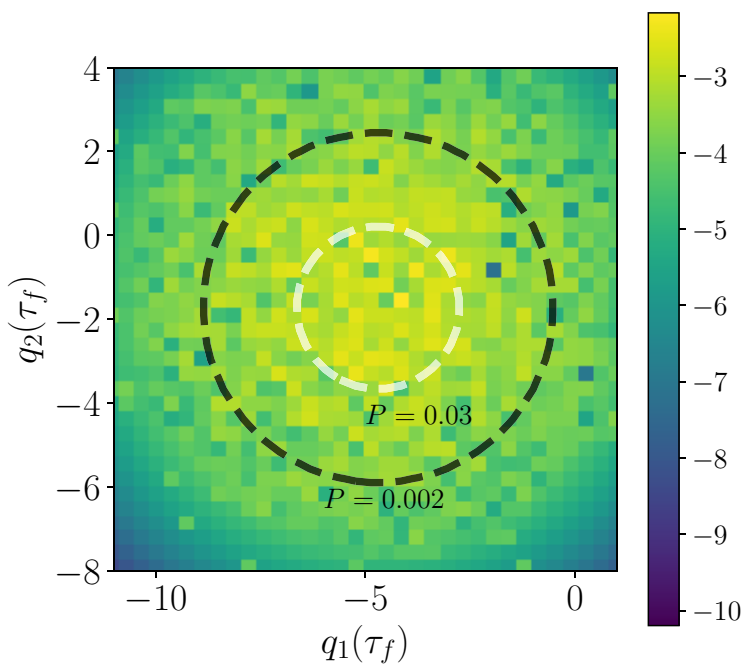

FIG. 4. The magnitude of the difference (in log scale) between the analytical and simulated probability densities of the final state at $\tau_{f}=10.0$, shown on the quadrature space. We start from the initial conditions $q_{1}(0)=3, q_{2}(0)=4$ with collapse time scales $\tau_{1}=\tau_{2}=\mathcal{T}=1.0$. The analytical expression is Eq. (27), while the simulated results correspond to the probability densities calculated from 100000 stochastic trajectories. The two dashed circular contours with analytical probability densities $P=0.03$ and $P=0.002$ illustrate the shape of the distribution. The values from the analytical prediction are very close to the ones from the simulations, with their differences lying between 0.0068 and $10^{-7}$. The agreement with the simulations illustrated here confirms the validity of the path integral calculation shown in Ref. III and Appendix E.

\section{OPTIMAL-PATH ENERGY}

The solution given in Eq. (22) can also be used to understand the energetics of measuring the oscillator, opening up the possibility of its adaptation in making quantum measurement engines or refrigerators $[85,86]$ and for feedback cooling [44 49]. Using Eqs. (20) and (22), the expectation value of the mechanical energy of the oscillator on a general optimal path is

$$
\begin{aligned}
E_{M}(\tau)= & \frac{1}{2}+\frac{1}{2}\left\{\left[\mathcal{Q}_{1 f}+\frac{\tau}{\tau_{f}}\left(q_{1 f}-\mathcal{Q}_{1 f}\right)\right]^{2}\right. \\
& \left.+\left[\mathcal{Q}_{2 f}+\frac{\tau}{\tau_{f}}\left(q_{2 f}-\mathcal{Q}_{2 f}\right)\right]^{2}\right\} .
\end{aligned}
$$

The mechanical energy evolves quadratically in time. The change in energy of the system is provided by the measurement. For $q_{1 f}=\mathcal{Q}_{1 f}$ and $q_{2 f}=\mathcal{Q}_{2 f}$ (i.e., final points on globally most likely optimal path), the mechanical energy stays constant with time.

The expression given in Eq. (28) also restricts the values of the final mechanical energies attainable during the measurement process with a certain measurement final time $\tau_{f}$ and boundary conditions. To illustrate this effect, Fig. 5(a) 
shows the quadratures and mechanical energy as functions of time for a sample optimal path. The quadratures are sinusoidal, with a time-dependent amplitude. The mechanical energy is parabolic, i.e., measurement first takes away from, but then provides energy to, the system. However, for the conditions chosen, the mechanical energy can only take the values lying on the purple dot-dashed curve. The analytical expression of the mechanical energy given in Eq. (28) therefore helps us understand the energy exchange between the system and its environment along paths with various postselections.

Figure 5(b) shows the same optimal path in quadrature space. The change in amplitude due to diffusive dynamics leads to spiral OPs instead of the circular paths that we see in unitary dynamics. The distance from the origin determines the mechanical energy of the oscillator. Revisiting Fig. 5(a), we see a sample optimal path the mechanical energy of which first decreases and then increases, at different times. The stochastic energy $[8-10,14,16]$ is

$$
\begin{aligned}
E_{s}= & \frac{2 \mathcal{T}}{\tau_{f}^{2}}\left[\left(q_{1 f}-\mathcal{Q}_{1 f}\right)^{2}+\left(q_{2 f}-\mathcal{Q}_{2 f}\right)^{2}\right] \\
& +\frac{4 \mathcal{T}}{\tau_{f}}\left(q_{1 f} \mathcal{Q}_{2 f}-q_{2 f} \mathcal{Q}_{1 f}\right)-\frac{1}{2 \mathcal{T}},
\end{aligned}
$$

which is a constant of motion.

Although Eq. (28) tells us that the mechanical energy does not depend on the value of $\mathcal{T}$, the probability of achieving a final state (i.e., postselection) does. This implies that arbitrarily large changes in energy are possible but that larger changes correspond to rarer events. Therefore, both Eq. (28) and Eq. (27) come into play while designing quantum measurement engines or refrigerators based on this system [85,86], as these engines rely on taking energy from the measurement process. Conceptually, such measurement-driven engines function by the observer or controller taking on a role analogous to Maxwell's demon [87-93]; by collecting real-time information about fluctuations of the system and then taking conditional feedback actions, one may make advantageous use of those fluctuations, beyond what is allowed merely on average (with ignorance of particular measurement outcomes).

\section{A. Information and purification}

It is fruitful at this point to reconsider the measurement outcome statistics, as expressed by the approximate surprisal given in Eq. (16):

$$
\mathcal{G}(\mathbf{r}, \mathbf{q})=\underbrace{-\frac{1}{2 \tau_{1}}\left(r_{1}-q_{1}\right)^{2}-\frac{1}{2 \tau_{2}}\left(r_{2}-q_{2}\right)^{2}}_{\text {Gaussian readout }}-\underbrace{\mathrm{g}\left(q_{3}, q_{5}\right)}_{\text {Information }} .
$$
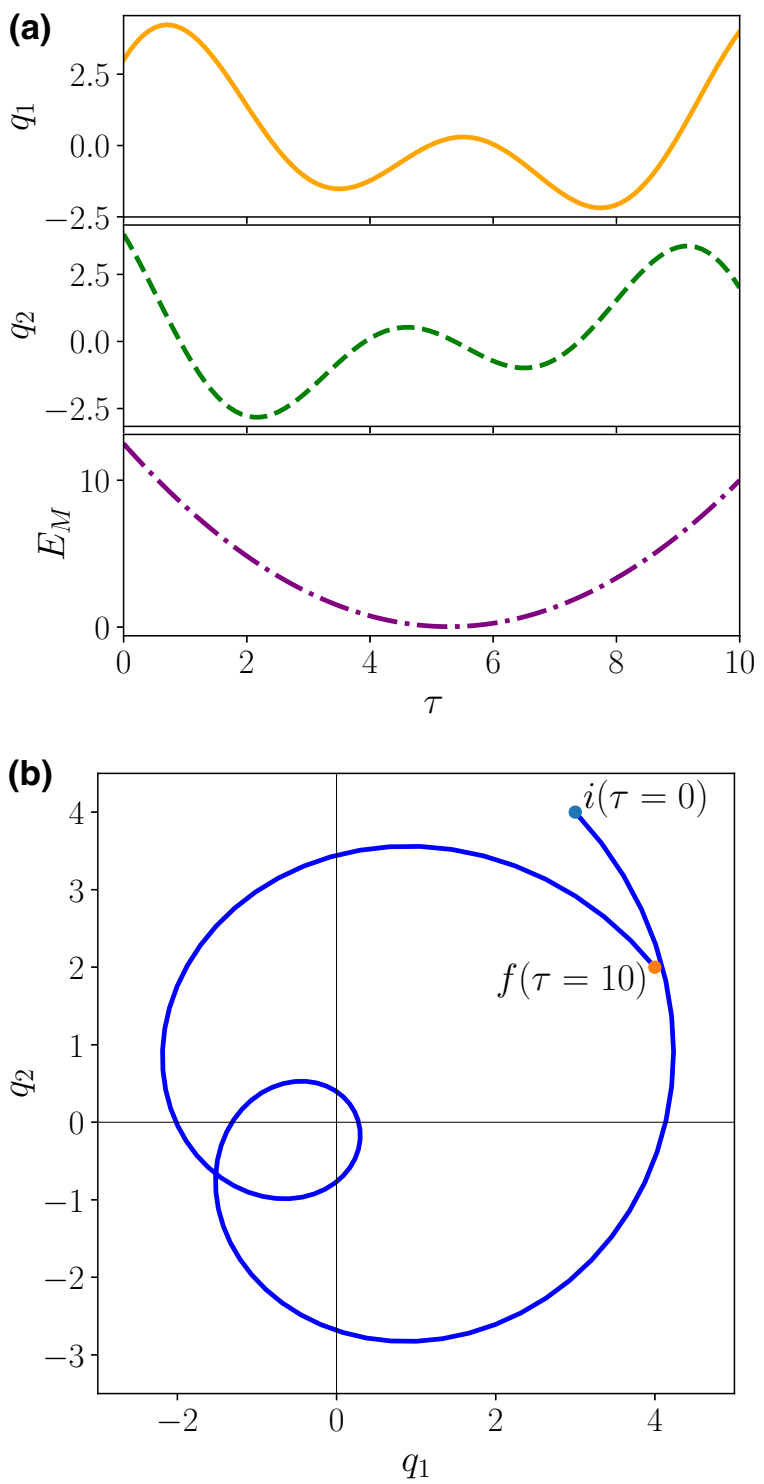

FIG. 5. The time evolution of the quadratures for boundary conditions $q_{1}(0)=3, q_{2}(0)=4, q_{1}\left(\tau_{f}=10\right)=4$, and $q_{2}\left(\tau_{f}=\right.$ 10) $=2$. (a) $q_{1}$ (top), $q_{2}$ (middle), and the mechanical energy $E_{M}$ (bottom panel) from Eq. (28) is shown for a sample optimal path for the equal-measurement-strength case $\left(\tau_{1}=\tau_{2}=\right.$ $\mathcal{T}=1$ ). The sinusoidal evolution of the quadratures is due to the unitary dynamics while the change in amplitude is due to the diffusive dynamics. (b) A plot of the sample optimal path in the quadrature space.

A straightforward application of Eq. (8) reveals that the mechanical energy evolves as

$$
\begin{aligned}
\dot{E}_{M} & =\mathbf{q} \cdot \dot{\mathbf{q}}=\mathbf{q}^{\top} \cdot \mathbf{\Omega} \cdot \mathbf{q}+\mathbf{q}^{\top} \cdot \mathbf{b} \cdot(\mathbf{R}-\mathbf{X}) \\
& =\frac{r_{1}-q_{1}}{2 \tau_{1}}\left(q_{1} q_{3}+q_{2} q_{4}\right)+\frac{r_{2}-q_{2}}{2 \tau_{2}}\left(q_{1} q_{4}+q_{2} q_{3}\right) .
\end{aligned}
$$


Clearly, then, the Gaussian fluctuations in the readouts $r_{1}$ and $r_{2}$ (the first two terms of $\mathcal{G}$ ) map directly onto the conditional shifts in the average mechanical energy.

It furthermore turns out that the information gain rate $\mathrm{g}\left(q_{3}, q_{5}\right)$ is intimately connected with the uncertainty in the mechanical energy. As detailed in Appendix B, the uncertainty area $A$ in phase space within one standard deviation of the Gaussian-state centroid $\left(q_{1}, q_{2}\right)$ is given by the determinant of the covariance matrix, such that

$$
A=q_{3} q_{5}-q_{4}^{2} \geq 1 .
$$

The stipulation $A \geq 1$ is a direct articulation of the Heisenberg uncertainty principle. For ideal measurements, the evolution of the covariance matrix given in Eq. (11) leads to a monotonic decrease of $A$ according to

$$
\dot{A}=-\left(\frac{q_{3}}{2 \tau_{1}}+\frac{q_{5}}{2 \tau_{2}}\right)(A-1)=-2 \mathrm{~g}\left(q_{3}, q_{5}\right)(A-1) .
$$

This can be understood as purification of the state via measurement with rate $2 \mathrm{~g}$, as information is collected; as mixed (impure) Gaussian states are an average over many pure Gaussian states, we may understand that the rate at which information as acquired, defined by $\mathrm{g}\left(q_{3}, q_{5}\right)$, also sets the rate at which our confidence of having an energy uncertainty saturating the Heisenberg uncertainty limit increases. Alternately: information gain is directly connected to the rate at which we may identify a microstate with a well-defined energy, to within the limits allowed by quantum mechanics, through time-continuous quadrature measurements. In this sense, $\mathrm{g}\left(q_{3}, q_{5}\right)$ defines the rate at which we eliminate any classical ignorance about the energy, in an impure Gaussian state, layered atop the Heisenberg-limited quantum uncertainty saturated by pure states. This can be understood in connection with the numerous works on rapid state purification (see, e.g., Refs. $[94,95])$.

Related comments are made by Belenchia et al. [25], who describe the evolution of the Wigner entropy $\mathcal{E}$ under continuous monitoring. This entropy is also defined in terms of the determinant of the covariance matrix, such that $\mathcal{E}=\frac{1}{2} \ln A$ and

$$
\dot{\mathcal{E}}=\mathrm{g}\left(q_{3}, q_{5}\right)\left(\frac{1}{A}-1\right)
$$

in our notation. The insights that we detail above and other recent independently derived results [96] reinforce each other. In summary, the log probability of the measurement statistics $\mathcal{G}(\mathbf{r}, \mathbf{q})$ that appears in the CDJ path integral is shown here to offer significant insights into the energetics of continuous monitoring for Gaussian measurements and dynamics. In particular, we illustrate how it characterizes both Gaussian energy fluctuations and information acquisition leading to state purification; together, these are powerful tools by which to understand and manipulate the energy of quantum systems under conditional dynamics, a topic of considerable recent interest in the literature [91-93].

\section{CONNECTION BETWEEN OPTIMAL PATH AND STOCHASTIC TRAJECTORIES}

Optimal paths for the Gaussian states can be defined as the most likely trajectory connecting a pair of boundary conditions. A complementary way to look at this is to postselect stochastic trajectories with particular boundary conditions and average the subset of them that are closest to each other, since we expect the trajectories to follow the MLP closely.

Following the method laid out in Refs. [11,15], we postselect 500 simulated trajectories according to the given boundary conditions. We then identify the subset of 50 trajectories that have the least distance between each other over their evolution. Such a grouping identifies a cluster of paths, the centroid of which is approximated by averaging the clustered trajectories. Figure 6 shows a comparison between these MLPs extracted from simulated data and the analytical MLP given in Eq. (22), for three different boundary conditions. The time dependence of $q_{1}$ and $q_{2}$ and the trajectory in quadrature space match exactly. Figure 6(a) also shows histograms of the quadratures for 100000 trajectories at times $\tau=2,3$, and 5. The spread of the histograms limits the extent to which energy can be added or withdrawn from the system via diffusive measurements with significant probability in a certain amount of time.

Figure 6(b) shows the analytical solution of $p_{1}, p_{2}$ (momenta conjugate to $q_{1}$ and $q_{2}$ ) and $E_{M}$ as functions of time for the three different boundary conditions previously shown in Fig. 6(a). The mechanical energy increases or decreases due to measurement for the choice of boundary conditions. As the sample boundary conditions $\mathbf{q}_{f}$ are very close to $\mathcal{Q}_{f}$, the energy changes are almost linear in time.

The quantum trajectories are described here by stochastic differential equations, whereas coupled ordinary differential equations characterize the optimal paths for the system. However, the above findings suggest that the optimal paths themselves provide an adequate effective description of a continuously monitored oscillator. In particular, from Eq. (27), we can see that with weaker measurements, the trajectories tend to be less diffusive. Therefore, instead of performing a computationally intensive simulation of a large number of such trajectories, the system dynamics can be effectively specified by a single optimal path.

\section{DISCUSSION}

We generalize the CDJ path integral formalism [8-10] to harmonic oscillators in general Gaussian states undergoing 
(a)

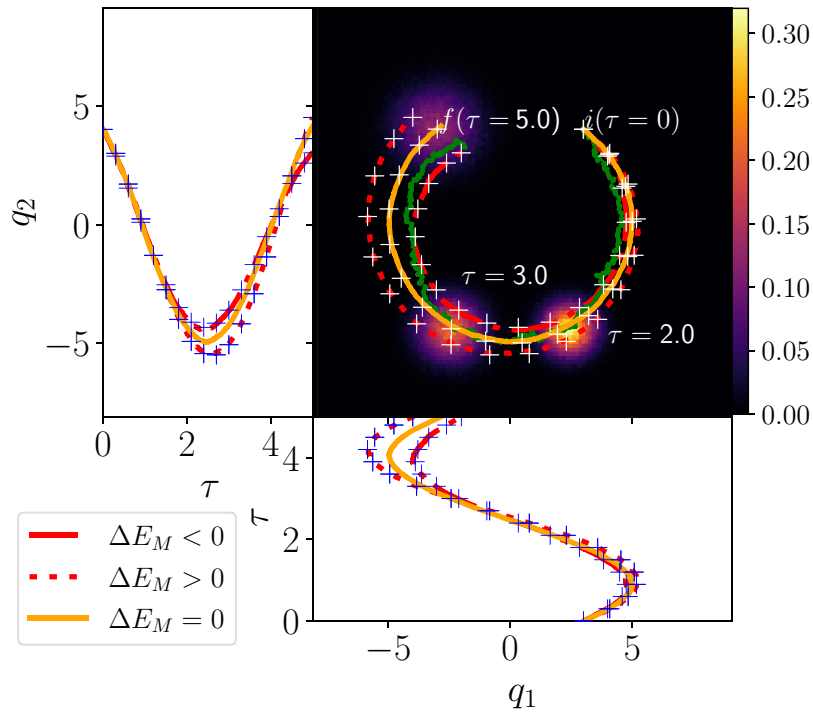

(b)

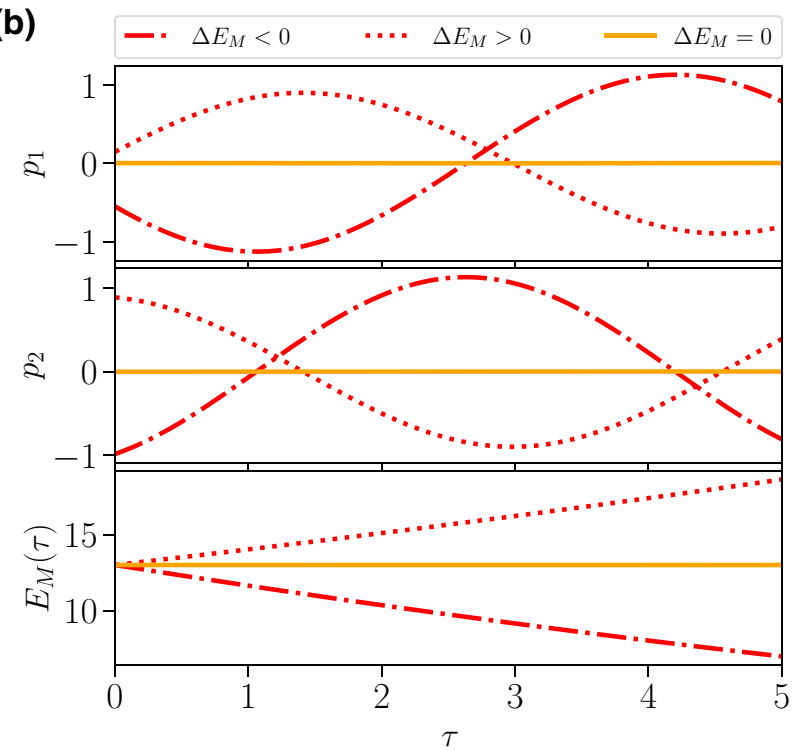

FIG. 6. (a) The evolution of trajectories and optimal paths starting from $q_{1}(0)=3, q_{2}(0)=4$ for $\tau_{1}=\tau_{2}=\mathcal{T}=1.0$ up to $\tau_{f}=5$. Note that $\tau=2 \pi$ corresponds to a full energy-conserving cycle. The yellow solid line corresponds to the average of 50 clustered trajectories for end points on the $p_{1}=p_{2}=0$ circle $\left[q_{1}(5.0)=-2.98, q_{2}(5.0)=4.01\right]$. The red dotted line corresponds to the average of 50 trajectories that lead to an increase in the mechanical energy of the system $\left[q_{1}(5.0)=-4.00, q_{2}(5.0)=4.50\right]$. The red dotdashed line is the average of trajectories with a decrease in the mechanical energy of the system $\left[q_{1}(5.0)=-2.00, q_{2}(5.0)=3.00\right]$. In the top-right panel, we show the optimal paths in quadrature space. The white "+" shows the analytical solutions corresponding to the three boundary conditions mentioned and the green line is a sample stochastic trajectory. Three histograms of the quadratures for three different times ( $\tau=2.0, \tau=3.0$, and $\tau=5.0$ superimposed) are also shown, along with a color bar on the right depicting the probability density values (see Sec. III) calculated from the simulations. The histograms show diffusion of the trajectories starting from the same initial point. The individual trajectories are not smooth but their postselected clustered averages give us the smooth analytical optimal paths exactly. In the top-left and bottom panels, we compare the time dependence of individual quadratures between the averaged stochastic trajectories and the analytical solution of the three optimal paths (the blue "+"). The analytical time dependence shows excellent agreement with the averaged results, supporting our interpretation of the optimal path. (b) The time evolution of the conjugate momenta and the mechanical energy for the three optimal paths shown in (a). The top two panels show $p_{1}$ and $p_{2}$ to be both sinusoidal for $\Delta E_{M} \neq 0$ and 0 for the energy-conserving path. The bottom-most panel shows $E_{M}$ to be increasing, decreasing, or constant depending on the optimal path.

continuous and weak simultaneous position and momentum measurements. The characteristic function gives us the evolution equations for the expectation values of position and momentum and the covariance-matrix elements. While the first two evolve stochastically, the covariance-matrix elements follow a set of three deterministic self-contained ordinary differential equations, describing purification of the state by measurement. Using the CDJ stochastic path integral formalism, we find probabilities for diffusive trajectories of the system in terms of a stochastic action. The most likely paths, found from extremizing the action, take the form of a resonantly driven oscillator. This leads to the change in amplitude and therefore mechanical energy of the oscillation due to measurement. The optimal-path description and the time-dependent expression of the mechanical energy have promising applicability for designing feedback control of quantum oscillators. For example, the extraction of energy through measurement for charging a quantum battery [97] or the cooling of mechanical resonators [44] provide exciting avenues for the incorporation of our results. For this particular system, we also successfully establish the interpretation of optimal paths as the average of clustered postselected stochastic trajectories.

In this work, we are able to evaluate the final-state probability density under continuous measurements by making exact functional integrals of the stochastic path integral. For the equal-measurement-strength case, this probability density is Gaussian centered around the globally most likely path. Generalizations of all results to unequal measurement strength, including position-only measurement, are given in the appendices. Although restricted to Gaussian states, these results can be useful for a vast range of experiments due to the ubiquity of such states.

Throughout our analysis, we assume time-independent strengths for the position and momentum measurements. The case of time-varying measurement strengths deserves closer inspection in the future as it can be used for, e.g., controlled squeezing. Generalization of the results to more than one dimension, or to coupled harmonic oscillators, 
has potential in the development of new avenues for entanglement generation between macroscopic oscillators $[13,51]$. With increasing dimensionality, finding a collection of stochastic trajectories would be more computationally expensive and might require a numerical method different from the Euler scheme used in most stochastic simulations. In such cases, our optimal-path description is again expected to provide a substantially concise narrative. Another natural extension includes applying the CDJ stochastic path integral formalism to general non-Gaussian states. Depending on the chosen state representation, such generalization might need a field-theory-based description. Although we do not see multipaths [14-16] in our system, it is possible that such phenomena might appear for non-Gaussian Schrödinger-cat states [98]. Generically, any system or class of states for which we may parametrize the dynamics with a manageable number of coordinates should be expected to be approachable via the same theoretical analysis as employed here. One could imagine, e.g., applying the present methods to monitoring Gottesman-KitaevPreskill (grid) states or Gaussian-state fermionic systems, where the stochastic path integral approach might lead to interesting insights. Therefore, our analysis offers new avenues for future developments on quantum trajectories of continuous-variable systems.

\section{ACKNOWLEDGMENTS}

We thank Sreenath Manikandan for providing insight throughout the project. This work has been supported by National Science Foundation (NSF) Grant No. DMR1809343, U.S. Army Research Office Grant No. W911NF18-10178, and John Templeton Foundation Grant No. 61835 .

\section{APPENDIX A: GAUSSIAN STATES}

The characteristic function of the density matrix $\hat{\rho}$ is defined as [77]

$$
\chi(\boldsymbol{\xi})=\operatorname{Tr}\left(\rho e^{i \boldsymbol{\xi}^{\top} \cdot \hat{\mathcal{R}}}\right) .
$$

Here, $\hat{\mathcal{R}}$ is a vector of operators given by $(\hat{X}, \hat{P})^{\top}$ and $\boldsymbol{\xi}$ is a vector in $\mathbb{R}^{2}$. For a Gaussian state, the characteristic function takes the form [77]

$$
\chi(\xi)=\exp \left(-\frac{1}{4} \boldsymbol{\xi}^{\top} \cdot \Gamma \cdot \boldsymbol{\xi}+i \boldsymbol{\mu}^{\top} \cdot \boldsymbol{\xi}\right) .
$$

$\Gamma$ is a $2 \times 2$ positive-definite real symmetric matrix [99], also known as the covariance matrix:

$$
\Gamma=\left(\begin{array}{ll}
q_{3} & q_{4} \\
q_{4} & q_{5}
\end{array}\right)
$$

and real column vector $\boldsymbol{\mu}=\left(q_{1}, q_{2}\right)^{\top}$. A Gaussian state is characterized completely by the matrices $\Gamma$ and $\mu$. For pure states, det $\Gamma=1$.

\section{APPENDIX B: COVARIANCE-MATRIX EVOLUTION}

The three equations of Eq. (11) give the deterministic evolution of the covariance-matrix elements. For all values of $\tau_{1}$ and $\tau_{2}$, they have one and only fixed point as given in Eq. (13a). We now prove that the solutions to these three equations for any initial values of covariance-matrix elements tend to the fixed points for $\tau \rightarrow \infty$.

Consider the determinant of the covariance matrix given in Eq. (A3), $A=q_{3} q_{5}-q_{4}^{2}$. Now, using the Heisenberg uncertainty relation $[100],\left\langle(\Delta \hat{X})^{2}\right\rangle\left\langle(\Delta \hat{P})^{2}\right\rangle$,

$$
\geq \frac{1}{4}|\langle[\Delta \hat{X}, \Delta \hat{P}]\rangle|^{2}+\frac{1}{4}|\langle\{\Delta \hat{X}, \Delta \hat{P}\}\rangle|^{2}
$$

leads to

$$
A=q_{3} q_{5}-q_{4}^{2} \geq 1
$$

From Eq. (11), we obtain

$$
\frac{d A}{d \tau}=-\left(\frac{q_{3}}{2 \tau_{1}}+\frac{q_{5}}{2 \tau_{2}}\right)(A-1)=-2 \mathrm{~g}(A-1) .
$$

As $q_{3}$ and $q_{5}$ are non-negative, Eqs. (B2) and (B3) show that the determinant is always decreasing with time. Integration of Eq. (B3) gives

$$
\ln \left|\frac{A-1}{A_{i}-1}\right|=-\int_{0}^{\tau}\left(\frac{q_{3}}{2 \tau_{1}}+\frac{q_{5}}{2 \tau_{2}}\right) d \tau^{\prime} .
$$

Using an inequality between the arithmetic and the geometric mean,

$$
\frac{q_{3}}{2 \tau_{1}}+\frac{q_{5}}{2 \tau_{2}} \geq \sqrt{\frac{q_{3} q_{5}}{\tau_{1} \tau_{2}}} \geq \sqrt{\frac{1}{\tau_{1} \tau_{2}}},
$$

we find that

$$
\left|\frac{A-1}{A_{i}-1}\right| \leq \exp \left(-\frac{\tau}{\sqrt{\tau_{1} \tau_{2}}}\right)
$$

Therefore, $A \rightarrow 1$ as $\tau \rightarrow \infty$ for any valid $A_{i}$, implying that the system goes toward a pure states for timecontinuous weak simultaneous position-momentum measurements. We assume $A_{i}$ to be the determinant value $(\geq 1)$ at $\tau=0$. If $A_{i}=1$, the determinant stays at 1 for all $\tau$. 
Now consider the variables $x, y$, and $z$, defined as follows:

$$
q_{3}=\tilde{q}_{3}+x, q_{4}=\tilde{q}_{4}+y, q_{5}=\tilde{q}_{5}+z .
$$

From Eq. (11), the evolution of these variables can be determined:

$$
\begin{aligned}
\frac{d x}{d \tau}= & -\frac{\tilde{q}_{3}}{\tau_{1}} x+\left(2-\frac{\tilde{q}_{4}}{\tau_{2}}\right) y-\left(\frac{x^{2}}{2 \tau_{1}}+\frac{y^{2}}{2 \tau_{2}}\right), \\
\frac{d y}{d \tau}= & -\left(1+\frac{\tilde{q}_{4}}{2 \tau_{1}}\right) x-\left(\frac{\tilde{q}_{3}}{2 \tau_{1}}+\frac{\tilde{q}_{5}}{2 \tau_{2}}\right) \\
& +\left(1-\frac{\tilde{q}_{4}}{2 \tau_{2}}\right) z-\left(\frac{x y}{2 \tau_{1}}+\frac{y z}{2 \tau_{2}}\right), \\
\frac{d z}{d \tau}= & -\left(2+\frac{\tilde{q}_{4}}{\tau_{1}}\right) y-\frac{\tilde{q}_{5}}{\tau_{2}} z-\left(\frac{y^{2}}{2 \tau_{1}}+\frac{z^{2}}{2 \tau_{2}}\right) .
\end{aligned}
$$

As $A \rightarrow 1$ for $\tau \rightarrow \infty$,

$$
\lim _{t \rightarrow \infty}\left(\tilde{q}_{5} x-2 \tilde{q}_{4} y+\tilde{q}_{3} z+x z-y^{2}\right)=0 .
$$

Eq. (B8) gives

$$
\frac{d}{d \tau}\left(x z-y^{2}\right)=-\frac{1}{2}\left(\frac{2 \tilde{q}_{3}+x}{\tau_{1}}+\frac{2 \tilde{q}_{5}+z}{\tau_{2}}\right)\left(x z-y^{2}\right) .
$$

Integrating yields

$$
\left|\frac{x z-y^{2}}{x_{i} z_{i}-y_{i}^{2}}\right|=\exp \left[-\int_{0}^{\tau} \frac{1}{2}\left(\frac{\tilde{q}_{3}+q_{3}}{\tau_{1}}+\frac{\tilde{q}_{5}+q_{5}}{\tau_{2}}\right) d \tau^{\prime}\right] .
$$

The denominator on the right-hand side corresponds to the initial values. Again applying the inequality between the arithmetic and geometric mean, we obtain

$$
\left|\frac{x z-y^{2}}{x_{i} z_{i}-y_{i}^{2}}\right| \leq \exp \left(-\sqrt{\frac{2}{\tau_{1} \tau_{2}}} \tau\right) .
$$

While Eq. (B6) gives Eq. (B9), Eq. (B12) proves that the nonlinear part of Eq. (B9), i.e., $x z-y^{2}$, also goes to 0 . We denote the $\tau \rightarrow \infty$ limits of $x, y$, and $z$ by $x_{f}, y_{f}$, and $z_{f}$, respectively. From Eq. (B9) and the fact that $\tilde{q}_{3} \tilde{q}_{5}-\tilde{q}_{4}^{2}=$ 1 , we obtain

$$
\left(\tilde{q}_{5} x_{f}-\tilde{q}_{3} z_{f}\right)^{2}+4 y_{f}^{2}=0 .
$$

Equations (B13) and (B9) give $x_{f}, y_{f}, z_{f}=0$. Hence, we provide a proof for the statement that at $\tau \rightarrow \infty$, the values of $q_{3}, q_{4}$, and $q_{5}$ tend to the fixed points of Eq. (11).

\section{APPENDIX C: PROPERTIES OF THE FIXED POINT}

We can linearize Eq. (B8) around the fixed point to obtain the following:

$$
\frac{d}{d \tau}\left(\begin{array}{l}
x \\
y \\
z
\end{array}\right)=\mathcal{M}\left(\begin{array}{l}
x \\
y \\
z
\end{array}\right)
$$

with the matrix

$$
\mathcal{M}=\left[\begin{array}{ccc}
-\frac{\tilde{q}_{3}}{\tau_{1}} & \left(2-\frac{\tilde{q}_{4}}{\tau_{2}}\right) & 0 \\
-\left(1+\frac{\tilde{q}_{4}}{2 \tau_{1}}\right) & -\left(\frac{\tilde{q}_{3}}{2 \tau_{1}}+\frac{\tilde{q}_{5}}{2 \tau_{2}}\right) & \left(1-\frac{\tilde{q}_{4}}{2 \tau_{2}}\right) \\
0 & -\left(2+\frac{\tilde{q}_{4}}{\tau_{1}}\right) & -\frac{\tilde{q}_{5}}{\tau_{2}}
\end{array}\right]
$$

The eigenvalues of the Eq. (C2) are

$$
\begin{aligned}
\lambda_{0} & =-\left(\frac{\tilde{q}_{3}}{2 \tau_{1}}+\frac{\tilde{q}_{5}}{2 \tau_{2}}\right), \\
\lambda_{+,-} & =-\left(\frac{\tilde{q}_{3}}{2 \tau_{1}}+\frac{\tilde{q}_{5}}{2 \tau_{2}}\right) \\
& \pm i \sqrt{\frac{1}{4}\left(\frac{\tilde{q}_{3}}{2 \tau_{1}}+\frac{\tilde{q}_{5}}{2 \tau_{2}}\right)^{2}+4-\frac{1}{\tau_{1} \tau_{2}} .}
\end{aligned}
$$

As the real parts of the eigenvalues are negative, the fixed point is stable (with a node in one direction and inward spirals along the other two directions).

\section{APPENDIX D: GENERAL SOLUTION FOR OPTIMAL PATH}

The analytical solution of Eq. (21) with the assumption of fixed-point (or steady-state) values of the covariancematrix elements and

$$
\mathbf{B}=\left(\begin{array}{ll}
\zeta & \Xi \\
\Xi & \Upsilon
\end{array}\right)
$$

for arbitrary measurement strengths gives

$$
\begin{aligned}
q_{1}(\tau)= & {\left[(\zeta+\Upsilon) \frac{\alpha_{1} \tau}{2}+q_{1 i}\right] \cos \tau } \\
& +\left\{(\zeta+\Upsilon) \frac{\alpha_{2} \tau}{2}+q_{2 i}-\left[(\Upsilon-\zeta) \frac{\alpha_{1}}{2}-\Xi \alpha_{2}\right]\right\} \\
& \sin \tau, \\
q_{2}(\tau)= & {\left[(\zeta+\Upsilon) \frac{\alpha_{2} \tau}{2}+q_{2 i}\right] \cos \tau } \\
& -\left\{(\zeta+\Upsilon) \frac{\alpha_{1} \tau}{2}+q_{1 i}-\left[(\Upsilon-\zeta) \frac{\alpha_{2}}{2}+\Xi \alpha_{1}\right]\right\} \\
& \sin \tau, \\
p_{1}(\tau)= & \alpha_{1} \cos \tau+\alpha_{2} \sin \tau, \quad p_{2}(\tau)=-\alpha_{1} \sin \tau \\
& +\alpha_{2} \cos \tau,
\end{aligned}
$$


where $\alpha_{1}$ and $\alpha_{2}$ are integration constants and $q_{1 i}$ and $q_{2 i}$ are initial values of $q_{1}$ and $q_{2}$. The values of $\alpha$ can be determined from the boundary conditions through the matrix equation

$$
\mathcal{A}\left(\tau_{f}\right)\left(\begin{array}{l}
\alpha_{1} \\
\alpha_{2}
\end{array}\right)=\left(\begin{array}{l}
q_{1 f}-q_{1 i} \cos \tau_{f}-q_{2 i} \sin \tau_{f} \\
q_{2 f}+q_{1 i} \sin \tau_{f}-q_{2 i} \cos \tau_{f}
\end{array}\right) .
$$

The elements of the matrix $\mathcal{A}\left(t_{f}\right)$ are

$$
\begin{array}{ll}
\mathcal{A}_{11}\left(\tau_{f}\right)=(\zeta+\Upsilon) \frac{\tau_{f} \cos \tau_{f}}{2}-\frac{1}{2}(\Upsilon-\zeta) \sin \tau_{f}, & \mathcal{A}_{12}\left(\tau_{f}\right)=\left[(\zeta+\Upsilon) \frac{\tau_{f}}{2}+\Xi\right] \sin \tau_{f}, \\
\mathcal{A}_{21}\left(\tau_{f}\right)=\left[-(\zeta+\Upsilon) \frac{\tau_{f}}{2}+\Xi\right] \sin \tau_{f}, & \mathcal{A}_{22}\left(\tau_{f}\right)=(\zeta+\Upsilon) \frac{\tau_{f} \cos \tau_{f}}{2}+\frac{1}{2}(\Upsilon-\zeta) \sin \tau_{f} .
\end{array}
$$

The determinant of $\mathcal{A}\left(\tau_{f}\right)$ is

$$
\operatorname{det}\left[\mathcal{A}\left(\tau_{f}\right)\right]=(\zeta+\Upsilon)^{2} \frac{\tau_{f}^{2}}{4}-\left[\frac{(\Upsilon-\zeta)^{2}}{4}+\Xi^{2}\right] \sin ^{2} \tau_{f}
$$

Comparing the constant term and the coefficient of the sine term, we find that

$$
\begin{gathered}
\frac{(\zeta+\Upsilon)^{2}}{4}-\frac{(\Upsilon-\zeta)^{2}}{4}-\Xi^{2}=\zeta \Upsilon-\Xi^{2} \\
=\frac{1}{16 \tau_{1} \tau_{2}}\left(\tilde{q}_{3} \tilde{q}_{5}-\tilde{q}_{4}^{2}\right)^{2}=\frac{1}{16 \tau_{1} \tau_{2}}>0
\end{gathered}
$$

From Eq. (D5), we find that

$$
\operatorname{det}\left[\mathcal{A}\left(\tau_{f}\right)\right]>\frac{(\zeta+\Upsilon)^{2}}{4}\left(\tau_{f}^{2}-\sin ^{2} \tau_{f}\right) \geq 0 .
$$

Hence, the matrix $\mathcal{A}\left(\tau_{f}\right)$ is always invertible, giving unique solutions for Eq. (21). Consequently, multipaths [14-16] do not exist in the present case.

Using Eqs. (D2) and (20), we can obtain the stochastic energy (a constant of motion):

$$
\begin{aligned}
E_{s}= & \frac{1}{2}\left(\zeta \alpha_{1}^{2}+2 \Xi \alpha_{1} \alpha_{2}+\Upsilon \alpha_{2}^{2}\right)+\left(q_{2 i} \alpha_{1}-q_{1 i} \alpha_{2}\right) \\
& -\frac{\tilde{q}_{3}}{4 \tau_{1}}-\frac{\tilde{q}_{5}}{4 \tau_{2}} .
\end{aligned}
$$

The expectation value of the mechanical energy is

$$
E_{M}(\tau)=\frac{1}{4}\left(\tilde{q}_{5}+\tilde{q}_{3}\right)+\frac{1}{2}\left[q_{1}(\tau)^{2}+q_{2}(\tau)^{2}\right] .
$$

\section{APPENDIX E: PROBABILITY DENSITY}

Here, we carry out an explicit calculation of the probability density $P\left(\mathbf{q}_{f} \mid \mathbf{q}_{i}\right)$. From Eqs. (24) and (18), we can write

$$
P\left(\mathbf{q}_{f} \mid \mathbf{q}_{i}\right)=\int \mathcal{D} \mathbf{p} \mathcal{D} \mathbf{q} \mathcal{D} \mathbf{r} \exp \left\{\int_{0}^{\tau_{f}} d \tau\left[-\mathbf{p} \cdot \dot{\mathbf{q}}+\mathbf{p}^{\top} \cdot(\boldsymbol{\Omega} \cdot \mathbf{q}+\mathbf{b} \cdot[\mathbf{R}-\mathbf{X}])-\frac{1}{2}(\mathbf{R}-\mathbf{X})^{2}-\mathbf{g}\right]\right\} .
$$

We note that for the $\mathbf{q}$ integral, we choose functions such that $\mathbf{q}(0)=\mathbf{q}_{i}$ and $\mathbf{q}\left(\tau_{f}\right)=\mathbf{q}_{f}$, while no such restrictions apply for the $\mathbf{p}$ or $\mathbf{r}$ integrals. We expect this integral to have the form

$$
P\left(\mathbf{q}_{f} \mid \mathbf{q}_{i}\right)=\mathcal{N} f\left(\mathbf{q}_{f}, \mathbf{q}_{i}, \tau_{f}\right)
$$

The normalization constant $\mathcal{N}$ is dependent on the measurement strengths and final time $\tau_{f}$. The functional integral with respect to the readouts is Gaussian and can be evaluated to give

$$
P\left(\mathbf{q}_{f} \mid \mathbf{q}_{i}\right)=\mathcal{N}_{1} \int \mathcal{D} \mathbf{p} \mathcal{D} \mathbf{q} \exp \left[\int_{0}^{\tau_{f}} d \tau\left(-\mathbf{p} \cdot \dot{\mathbf{q}}+\frac{1}{2} \mathbf{p}^{\top} \cdot \mathbf{B} \cdot \mathbf{p}+\mathbf{p}^{\top} \cdot \boldsymbol{\Omega} \cdot \mathbf{q}-\mathbf{g}\right)\right]
$$


with $\mathcal{N}_{1}$ as a constant dependent on $\tau_{f}$. We now expand the quadratures and their conjugate momenta around the $\mathrm{OP}$ solution $\mathbf{q}=\mathbf{q O P}(\tau)+\delta \mathbf{q}$ and $\mathbf{p}=\mathbf{p} \mathrm{OP}(\tau)+\delta \mathbf{p} . \mathbf{q O P}(\tau)$ and $\operatorname{pop}(\tau)$ satisfy Eq. (21) with boundary conditions $\mathbf{q O P}(0)=\mathbf{q}_{i}$, and $\mathbf{q O P}\left(\tau_{f}\right)=\mathbf{q}_{f}$. This implies that $\delta \mathbf{q}(0)=$ $\delta \mathbf{q}\left(\tau_{f}\right)=0$, with no restrictions on $\delta \mathbf{p}$. Using the definitions of $\mathbf{B}, \boldsymbol{\Omega}$, Eq. (21), and boundary conditions, Eq. (E3) can be simplified to

$$
\begin{aligned}
P\left(\mathbf{q}_{f} \mid \mathbf{q}_{i}\right)= & \mathcal{N}_{1} \int \mathcal{D} \delta \mathbf{p} \mathcal{D} \delta \mathbf{q} \exp \left[\int_{0}^{\tau_{f}} d \tau\left(-\delta \mathbf{p} \cdot \delta \dot{\mathbf{q}}+\frac{1}{2} \delta \mathbf{p}^{\top} \cdot \mathbf{B} \cdot \delta \mathbf{p}+\delta \mathbf{p}^{\top} \cdot \mathbf{\Omega} \cdot \delta \mathbf{q}\right)\right] \\
& \times \exp \left[\int_{0}^{\tau_{f}} d \tau\left(-\frac{1}{2} \mathbf{p}_{\mathrm{OP}}^{\top} \cdot \mathbf{B} \cdot \mathbf{p o P}_{\mathrm{OP}}-\mathrm{g}\right)\right] .
\end{aligned}
$$

Except for $\mathbf{p}_{\mathrm{OP}}^{\top} \cdot \mathbf{B} \cdot \mathbf{p}_{\mathrm{OP}}$, everything else in the exponential can be absorbed in the normalization constant, giving

$$
P\left(\mathbf{q}_{f} \mid \mathbf{q}_{i}\right)=\mathcal{N} \exp \left[-\frac{1}{2} \int_{0}^{\tau_{f}} d \tau \mathbf{p}_{\mathrm{OP}}^{\top} \cdot \mathbf{B} \cdot \mathbf{p}_{\mathrm{OP}}\right] .
$$

For the steady-state case (i.e., fixed-point values of $q_{3}, q_{4}$, and $q_{5}$ ), from Eqs. (D2) and (D3), the OP solutions for the momenta are $\mathbf{p}_{\mathrm{OP}}=R(\tau) \mathcal{A}\left(\tau_{f}\right)^{-1} \Delta \mathbf{Q}$, with $R(\tau)$ as a rotation matrix and $\Delta \mathbf{Q}=\left(\Delta Q_{1}, \Delta Q_{2}\right)^{\top}=\left(q_{1 f}-\mathcal{Q}_{1 f}, q_{2 f}-\right.$ $\left.\mathcal{Q}_{2 f}\right)^{\top}$. After the time integration in Eq. (E5) and calculation of the normalization constant, the probability density becomes

$$
P\left(\mathbf{q}_{f} \mid \mathbf{q}_{i}\right)=\frac{\sqrt{\operatorname{det}(\Sigma)}}{2 \pi} \exp \left[-\frac{1}{2} \Delta \mathbf{Q}^{\top} \cdot \Sigma \cdot \Delta \mathbf{Q}\right]
$$

with the matrix

$$
\Sigma=\left(\mathcal{A}\left(\tau_{f}\right)^{\top}\right)^{-1}\left\{\frac{\zeta+\Upsilon}{2} \tau_{f} \mathbb{1}+\frac{\sin \tau_{f}}{2}\left(\begin{array}{cc}
(\zeta-\Upsilon) \cos \tau_{f}-2 \Xi \sin \tau_{f} & (\zeta-\Upsilon) \sin \tau_{f}+2 \Xi \cos \tau_{f} \\
(\zeta-\Upsilon) \sin \tau_{f}+2 \Xi \cos \tau_{f} & -(\zeta-\Upsilon) \cos \tau_{f}+2 \Xi \sin \tau_{f}
\end{array}\right)\right\} \mathcal{A}\left(\tau_{f}\right)^{-1} .
$$

Figure 7 compares our analytical calculations with the simulations of 100000 trajectories. Our path integral approach correctly predicts the squeezing of the Gaussian probability density function for the general measurementstrength case. The probability densities of the individual final quadratures $q_{1 f}$ and $q_{2 f}$ become

$$
\begin{aligned}
& P\left(q_{1 f} \mid \mathbf{q}_{i}\right)=\sqrt{\frac{\operatorname{det}(\Sigma)}{2 \pi \Sigma_{22}}} \exp \left[-\frac{\operatorname{det}(\Sigma)}{2 \Sigma_{22}} \Delta Q_{1}^{2}\right] \\
& P\left(q_{2 f} \mid \mathbf{q}_{i}\right)=\sqrt{\frac{\operatorname{det}(\Sigma)}{2 \pi \Sigma_{11}}} \exp \left[-\frac{\operatorname{det}(\Sigma)}{2 \Sigma_{11}} \Delta Q_{2}^{2}\right]
\end{aligned}
$$

\section{APPENDIX F: POSITION MEASUREMENT}

For only position measurement [see Fig. 1(a)], $\tau_{1}=\mathcal{T}$ and $\tau_{2} \rightarrow \infty$. The fixed-point values of $q_{3}, q_{4}$, and $q_{5}$ are given by

$$
\tilde{q}_{3}=\sqrt{4 \mathcal{T}\left(\sqrt{1+4 \mathcal{T}^{2}}-2 \mathcal{T}\right)}
$$

$$
\begin{aligned}
& \tilde{q}_{4}=\sqrt{1+4 \mathcal{T}^{2}}-2 \mathcal{T} \\
& \tilde{q}_{5}=\sqrt{\frac{1}{\mathcal{T}}\left(\sqrt{1+4 \mathcal{T}^{2}}-2 \mathcal{T}\right)\left(1+4 \mathcal{T}^{2}\right)} .
\end{aligned}
$$

The solutions for the optimal path given in Eq. (D2) are

$$
\begin{aligned}
q_{1}(\tau)= & {\left[\frac{\alpha_{1}\left(\tilde{q}_{3}^{2}+\tilde{q}_{4}^{2}\right)}{8 \mathcal{T}} \tau+q_{1 i}\right] \cos \tau+\left[\frac{\alpha_{2}\left(\tilde{q}_{3}^{2}+\tilde{q}_{4}^{2}\right)}{8 \mathcal{T}} \tau\right.} \\
& \left.+q_{2 i}+\frac{\alpha_{1}\left(\tilde{q}_{3}^{2}-\tilde{q}_{4}^{2}\right)}{8 \mathcal{T}}+\frac{\tilde{q}_{3} \tilde{q}_{4} \alpha_{2}}{4 \mathcal{T}}\right] \sin \tau, \\
q_{2}(\tau)= & -\left[\frac{\alpha_{1}\left(\tilde{q}_{3}^{2}+\tilde{q}_{4}^{2}\right)}{8 \mathcal{T}} \tau+q_{1 i}+\frac{\alpha_{2}\left(\tilde{q}_{3}^{2}-\tilde{q}_{4}^{2}\right)}{8 \mathcal{T}}-\frac{\tilde{q}_{3} \tilde{q}_{4} \alpha_{1}}{4 \mathcal{T}}\right] \\
& \times \sin \tau+\left[\frac{\alpha_{2}\left(\tilde{q}_{3}^{2}+\tilde{q}_{4}^{2}\right)}{8 \mathcal{T}} \tau+q_{2 i}\right] \cos \tau, \\
p_{1}(\tau)= & \alpha_{1} \cos \tau+\alpha_{2} \sin \tau, \\
p_{2}(\tau)= & -\alpha_{1} \sin \tau+\alpha_{2} \cos \tau .
\end{aligned}
$$

Just as in the general case, $\alpha_{1}$ and $\alpha_{2}$ are integration constants and $q_{1 i}$ and $q_{2 i}$ are initial values of $q_{1}$ and $q_{2}$. 
The values of $\alpha$ can be determined from the final conditions through the matrix equation

$$
\mathcal{A}\left(\tau_{f}\right)\left(\begin{array}{l}
\alpha_{1} \\
\alpha_{2}
\end{array}\right)=\left[\begin{array}{l}
q_{1 f}-q_{1 i} \cos \tau_{f}-q_{2 i} \sin \tau_{f} \\
q_{2 f}+q_{1 i} \sin \tau_{f}-q_{2 i} \cos \tau_{f}
\end{array}\right] .
$$

The matrix $\mathcal{A}\left(\tau_{f}\right)$ is given by

$$
\mathcal{A}\left(\tau_{f}\right)=\frac{1}{8 \mathcal{T}}\left[\begin{array}{cc}
\left(\tilde{q}_{3}^{2}+\tilde{q}_{4}^{2}\right) \tau_{f} \cos \tau_{f}+\left(\tilde{q}_{3}^{2}-\tilde{q}_{4}^{2}\right) \sin \tau_{f} & \left(\left(\tilde{q}_{3}^{2}+\tilde{q}_{4}^{2}\right) \tau_{f}+2 \tilde{q}_{3} \tilde{q}_{4}\right) \sin \tau_{f} \\
\left(-\left(\tilde{q}_{3}^{2}+\tilde{q}_{4}^{2}\right) \tau_{f}+2 \tilde{q}_{3} \tilde{q}_{4}\right) \sin \tau_{f} & \left(\tilde{q}_{3}^{2}+\tilde{q}_{4}^{2}\right) \tau_{f} \cos \tau_{f}-\left(\tilde{q}_{3}^{2}-\tilde{q}_{4}^{2}\right) \sin \tau_{f}
\end{array}\right] .
$$

The determinant of Eq. (F4) is

$$
\operatorname{det}\left[\mathcal{A}\left(\tau_{f}\right)\right]=\frac{\left(\tilde{q}_{3}^{2}+\tilde{q}_{4}^{2}\right)^{2}}{64 \mathcal{T}^{2}}\left(\tau_{f}^{2}-\sin ^{2} \tau_{f}\right)>0 \quad\left(\forall \tau_{f}>0\right),
$$

again proving the nonexistence of multipaths.

\section{APPENDIX G: TRANSITORY DYNAMICS}

Throughout our optimal-path analysis, we assume that the covariance-matrix elements take their steady-state values [see Eq. (13a)]. This is done for simplicity and in order to understand cases admitting completely analytical

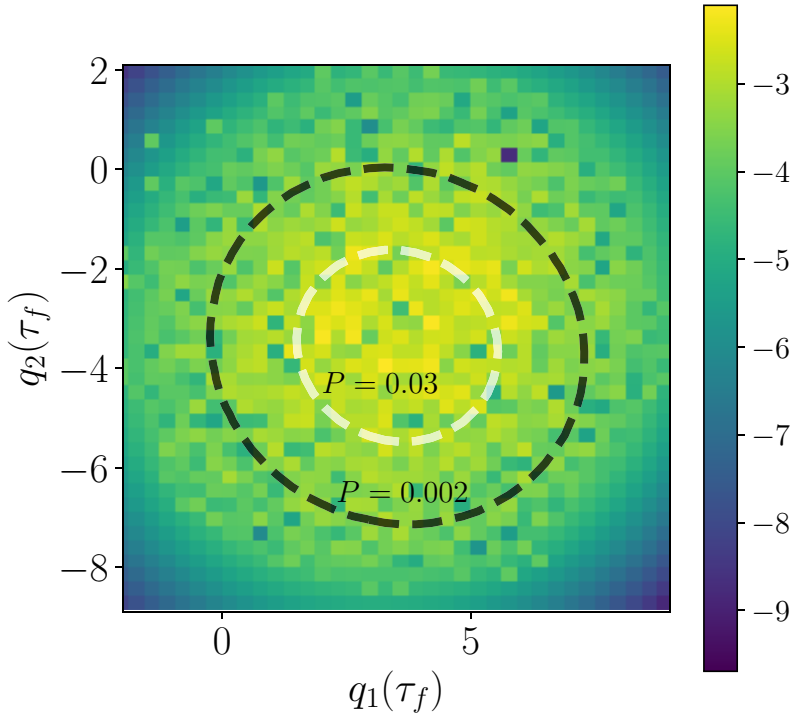

FIG. 7. Here, we show (in log scale) the magnitude of the difference between the simulated (100000 stochastic trajectories) and analytical [Eq. (E6)] final-state probability densities for $\tau_{f}=8.0$, starting from the initial state $q_{1}(0)=3$ and $q_{2}(0)=4$. Unlike Fig. 4, here we look at the case of unequal collapse time scales (or, equivalently, measurement strengths), arbitrarily chosen to have the values $\tau_{1}=0.7$ and $\tau_{2}=3.0$. In this case as well, our prediction using Eq. (E6) matches with the simulations. In contrast with Fig. 4, the two analytical-probability-density contours shown here are slightly squeezed. solutions. However, nothing in our approach is fundamentally limited to this case. Here, we comment on how the analysis changes when we take into account the temporal dynamics of $q_{3}, q_{4}$, and $q_{5}$. It can be seen that the form of the stochastic Hamiltonian [Eq. (18)], the optimal readouts [Eq. (19)], the optimal Hamiltonian [Eq. (20)], and the equations of motion for the optimal paths [Eq. (21)] remain the same. However, the diffusion matrices $\mathbf{b}$ and B now implicitly depend on time, as does the information gain rate g. It is clear from Eqs. (B2), (B3), and (B5) that $\mathrm{g}$ is larger for impure states and in some sense can be understood as a measure of the purifying power provided by our joint measurements. Although components of $\mathbf{p}$ retain their sinusoidal behavior, the components of $\mathbf{q}$ generally do not and may require numerical solutions in the transient regime. The probability density of final states also retains the form given in Eq. (E5). Since B is time dependent, the integral inside the exponential now generally needs to be performed numerically as well. Additionally, the normalization factor $\mathcal{N}$ depends in general on the measurement strengths, the final time, and the initial values of the covariance-matrix elements. As Fig. 2 suggests, the covariance-matrix elements reach their steady-state values in collapse time scales, making our steady-state assumption sufficient for cases where the oscillator period is longer than collapse time scales.

[1] H. J. Carmichael, An Open Systems Approach to Quantum Optics (Springer, Berlin, 1993).

[2] H. M. Wiseman and G. J. Milburn, Quantum Measurement and Control (Cambridge University Press, Cambridge, 2009).

[3] A. Barchielli and M. Gregoratti, Quantum Trajectories and Measurements in Continuous Time (Springer-Verlag, Berlin, 2009).

[4] K. Jacobs, Quantum Measurement Theory and its Applications (Cambridge University Press, Cambridge, 2014).

[5] J. Zhang, Y.-x. Liu, R.-B. Wu, K. Jacobs, and F. Nori, Quantum feedback: Theory, experiments, and applications, Phys. Rep. 679, 1 (2017). 
[6] A. N. Jordan, Quantum physics: Watching the wavefunction collapse, Nature 502, 177 (2013).

[7] M. Rossi, D. Mason, J. Chen, and A. Schliesser, Observing and Verifying the Quantum Trajectory of a Mechanical Resonator, Phys. Rev. Lett. 123, 163601 (2019).

[8] A. Chantasri, J. Dressel, and A. N. Jordan, Action principle for continuous quantum measurement, Phys. Rev. A 88, 042110 (2013).

[9] A. Chantasri and A. N. Jordan, Stochastic path-integral formalism for continuous quantum measurement, Phys. Rev. A 92, 032125 (2015).

[10] A. Chantasri, Stochastic Path Integral Formalism for Continuous Quantum Measurement, PhD Dissertation, University of Rochester (2016).

[11] S. J. Weber, A. Chantasri, J. Dressel, A. N. Jordan, K. W. Murch, and I. Siddiqi, Mapping the optimal route between two quantum states, Nature 511, 570 (2014).

[12] A. N. Jordan, A. Chantasri, P. Rouchon, and B. Huard, Anatomy of fluorescence: Quantum trajectory statistics from continuously measuring spontaneous emission, Quantum Stud.: Math. Found. 3, 237 (2016).

[13] A. Chantasri, M. E. Kimchi-Schwartz, N. Roch, I. Siddiqi, and A. N. Jordan, Quantum Trajectories and Their Statistics for Remotely Entangled Quantum Bits, Phys. Rev. X 6, 041052 (2016).

[14] P. Lewalle, A. Chantasri, and A. N. Jordan, Prediction and characterization of multiple extremal paths in continuously monitored qubits, Phys. Rev. A 95, 042126 (2017).

[15] M. Naghiloo, D. Tan, P. M. Harrington, P. Lewalle, A. N. Jordan, and K. W. Murch, Quantum caustics in resonancefluorescence trajectories, Phys. Rev. A 96, 053807 (2017).

[16] P. Lewalle, J. Steinmetz, and A. N. Jordan, Chaos in continuously monitored quantum systems: An optimal-path approach, Phys. Rev. A 98, 012141 (2018).

[17] P. Lewalle, S. K. Manikandan, C. Elouard, and A. N. Jordan, Measuring fluorescence to track a quantum emitter's state: A theory review, Contemp. Phys. 61, 26 (2020).

[18] M. B. Menskii, Decoherence and the theory of continuous quantum measurements, Phys.-Uspekhi 41, 923 (1998).

[19] M. B. Mensky, R. Onofrio, and C. Presilla, Continuous quantum monitoring of position of nonlinear oscillators, Phys. Lett. A 161, 236 (1991).

[20] C. Presilla, R. Onofrio, and U. Tambini, Measurement quantum mechanics and experiments on quantum Zeno effect, Ann. Phys. (N.Y.) 248, 95 (1996).

[21] H. Grabert, P. Schramm, and G.-L. Ingold, Quantum Brownian motion: The functional integral approach, Phys. Rep. 168, 115 (1988).

[22] L. Ferialdi and A. Bassi, Exact Solution for a NonMarkovian Dissipative Quantum Dynamics, Phys. Rev. Lett. 108, 170404 (2012).

[23] K. Jacobs and D. A. Steck, A straightforward introduction to continuous quantum measurement, Contemp. Phys. 47, 279 (2006).

[24] M. G. Genoni, L. Lami, and A. Serafini, Conditional and unconditional Gaussian quantum dynamics, Contemp. Phys. 57, 331 (2016).
[25] A. Belenchia, L. Mancino, G. T. Landi, and M. Paternostro, Entropy production in continuously measured Gaussian quantum systems, npj Quantum Inf. 6, 1 (2020).

[26] A. C. Doherty and K. Jacobs, Feedback control of quantum systems using continuous state estimation, Phys. Rev. A 60, 2700 (1999).

[27] I. Guevara and H. Wiseman, Quantum State Smoothing, Phys. Rev. Lett. 115, 180407 (2015).

[28] Z. Huang and M. Sarovar, Smoothing of Gaussian quantum dynamics for force detection, Phys. Rev. A 97, 042106 (2018).

[29] K. T. Laverick, A. Chantasri, and H. M. Wiseman, Quantum State Smoothing for Linear Gaussian Systems, Phys. Rev. Lett. 122, 190402 (2019).

[30] K. T. Laverick, A. Chantasri, and H. M. Wiseman, Linear Gaussian quantum state smoothing: How to optimally "unobserve" a quantum system (2020), ArXiv:2008.13348.

[31] K. T. Laverick, A. Chantasri, and H. M. Wiseman, General criteria for quantum state smoothing with necessary and sufficient criteria for linear Gaussian quantum systems, Quantum Stud.: Math. Found. 8, 37 (2021).

[32] K. T. Laverick, A. Chantasri, and H. M. Wiseman, Linear Gaussian quantum state smoothing: Understanding the optimal unravelings for Alice to estimate Bob's state, Phys. Rev. A 103, 012213 (2021).

[33] K. T. Laverick, A. Chantasri, and H. M. Wiseman, General Criteria for Quantum State Smoothing with Necessary and Sufficient Criteria for Linear Gaussian Quantum Systems, Quantum Stud.: Math. Found. (2020).

[34] M. Aspelmeyer, T. J. Kippenberg, and F. Marquardt, Cavity optomechanics, Rev. Mod. Phys. 86, 1391 (2014).

[35] A. A. Clerk, M. H. Devoret, S. M. Girvin, F. Marquardt, and R. J. Schoelkopf, Introduction to quantum noise, measurement, and amplification, Rev. Mod. Phys. 82, 1155 (2010).

[36] M. R. Vanner, I. Pikovski, and M. S. Kim, Towards optomechanical quantum state reconstruction of mechanical motion, Ann. Phys. 527, 15 (2015).

[37] A. H. Safavi-Naeini, J. Chan, J. T. Hill, T. P. Mayer Alegre, A. Krause, and O. Painter, Observation of Quantum Motion of a Nanomechanical Resonator, Phys. Rev. Lett. 108, 033602 (2012).

[38] C. F. Ockeloen-Korppi, E. Damskägg, J.-M. Pirkkalainen, A. A. Clerk, M. J. Woolley, and M. A. Sillanpää, Quantum Backaction Evading Measurement of Collective Mechanical Modes, Phys. Rev. Lett. 117, 140401 (2016).

[39] N. S. Kampel, R. W. Peterson, R. Fischer, P.-L. Yu, K. Cicak, R. W. Simmonds, K. W. Lehnert, and C. A. Regal, Improving Broadband Displacement Detection with Quantum Correlations, Phys. Rev. X 7, 021008 (2017).

[40] C. B. Møller, R. A. Thomas, G. Vasilakis, E. Zeuthen, Y. Tsaturyan, M. Balabas, K. Jensen, A. Schliesser, K. Hammerer, and E. S. Polzik, Quantum back-action-evading measurement of motion in a negative mass reference frame, Nature 547, 191 (2017).

[41] V. Sudhir, D. J. Wilson, R. Schilling, H. Schütz, S. A. Fedorov, A. H. Ghadimi, A. Nunnenkamp, and T. 
J. Kippenberg, Appearance and Disappearance of Quantum Correlations in Measurement-Based Feedback Control of a Mechanical Oscillator, Phys. Rev. X 7, 011001 (2017).

[42] T. P. Purdy, R. W. Peterson, and C. A. Regal, Observation of radiation pressure shot noise on a macroscopic object, Science 339, 801 (2013).

[43] J. D. Thompson, B. M. Zwickl, A. M. Jayich, F. Marquardt, S. M. Girvin, and J. G. E. Harris, Strong dispersive coupling of a high-finesse cavity to a micromechanical membrane, Nature 452, 72 (2008).

[44] M. Rossi, D. Mason, J. Chen, Y. Tsaturyan, and A. Schliesser, Measurement-based quantum control of mechanical motion, Nature 563, 53 (2018).

[45] D. J. Wilson, V. Sudhir, N. Piro, R. Schilling, A. Ghadimi, and T. J. Kippenberg, Measurement-based control of a mechanical oscillator at its thermal decoherence rate, Nature 524, 325 (2015).

[46] A. G. Krause, T. D. Blasius, and O. Painter, Optical Read Out and Feedback Cooling of a Nanostring Optomechanical Cavity (2015), ArXiv:1506.01249.

[47] M. Poggio, C. L. Degen, H. J. Mamin, and D. Rugar, Feedback Cooling of a Cantilever's Fundamental Mode below 5 mK, Phys. Rev. Lett. 99, 017201 (2007).

[48] D. Kleckner and D. Bouwmeester, Sub-kelvin optical cooling of a micromechanical resonator, Nature 444, 75 (2006).

[49] T. Corbitt, C. Wipf, T. Bodiya, D. Ottaway, D. Sigg, N. Smith, S. Whitcomb, and N. Mavalvala, Optical Dilution and Feedback Cooling of a Gram-Scale Oscillator to 6.9 mK, Phys. Rev. Lett. 99, 160801 (2007).

[50] R. Riedinger, A. Wallucks, I. Marinković, C. Löschnauer, M. Aspelmeyer, S. Hong, and S. Gröblacher, Remote quantum entanglement between two micromechanical oscillators, Nature 556, 473 (2018).

[51] C. F. Ockeloen-Korppi, E. Damskägg, J.-M. Pirkkalainen, M. Asjad, A. A. Clerk, F. Massel, M. J. Woolley, and M. A. Sillanpää, Stabilized entanglement of massive mechanical oscillators, Nature 556, 478 (2018).

[52] K. C. Lee, M. R. Sprague, B. J. Sussman, J. Nunn, N. K. Langford, X.-M. Jin, T. Champion, P. Michelberger, K. F. Reim, D. England, D. Jaksch, and I. A. Walmsley, Entangling macroscopic diamonds at room temperature, Science 334, 1253 (2011).

[53] R. Riedinger, S. Hong, R. A. Norte, J. A. Slater, J. Shang, A. G. Krause, V. Anant, M. Aspelmeyer, and S. Gröblacher, Non-classical correlations between single photons and phonons from a mechanical oscillator, Nature 530, 313 (2016).

[54] T. A. Palomaki, J. D. Teufel, R. W. Simmonds, and K. W. Lehnert, Entangling mechanical motion with microwave fields, Science 342, 710 (2013).

[55] M. Ringbauer, T. J. Weinhold, L. A. Howard, A. G. White, and M. R. Vanner, Generation of mechanical interference fringes by multi-photon counting, New J. Phys. 20, 053042 (2018).

[56] C. U. Lei, A. J. Weinstein, J. Suh, E. E. Wollman, A. Kronwald, F. Marquardt, A. A. Clerk, and K. C. Schwab, Quantum Nondemolition Measurement of a Quantum Squeezed
State Beyond the $3 \mathrm{~dB}$ Limit, Phys. Rev. Lett. 117, 100801 (2016).

[57] E. E. Wollman, C. U. Lei, A. J. Weinstein, J. Suh, A. Kronwald, F. Marquardt, A. A. Clerk, and K. C. Schwab, Quantum squeezing of motion in a mechanical resonator, Science 349, 952 (2015).

[58] J.-M. Pirkkalainen, E. Damskägg, M. Brandt, F. Massel, and M. A. Sillanpää, Squeezing of Quantum Noise of Motion in a Micromechanical Resonator, Phys. Rev. Lett. 115, 243601 (2015).

[59] F. Lecocq, J. B. Clark, R. W. Simmonds, J. Aumentado, and J. D. Teufel, Quantum Nondemolition Measurement of a Nonclassical State of a Massive Object, Phys. Rev. X 5, 041037 (2015).

[60] A. Pontin, M. Bonaldi, A. Borrielli, F. S. Cataliotti, F. Marino, G. A. Prodi, E. Serra, and F. Marin, Squeezing a Thermal Mechanical Oscillator by Stabilized Parametric Effect on the Optical Spring, Phys. Rev. Lett. 112, 023601 (2014).

[61] S. Hong, R. Riedinger, I. Marinković, A. Wallucks, S. G. Hofer, R. A. Norte, M. Aspelmeyer, and S. Gröblacher, Hanbury Brown and Twiss interferometry of single phonons from an optomechanical resonator, Science 358, 203 (2017).

[62] J. D. Cohen, S. M. Meenehan, G. S. MacCabe, S. Gröblacher, A. H. Safavi-Naeini, F. Marsili, M. D. Shaw, and $\mathrm{O}$. Painter, Phonon counting and intensity interferometry of a nanomechanical resonator, Nature 520, 522 (2015).

[63] W.-L. Ma, S. Puri, R. J. Schoelkopf, M. H. Devoret, S. M. Girvin, and L. Jiang, Quantum control of bosonic modes with superconducting circuits, Sci. Bull. 66, 1789 (2021).

[64] S. Hacohen-Gourgy, L. S. Martin, E. Flurin, V. V. Ramasesh, K. B. Whaley, and I. Siddiqi, Dynamics of simultaneously measured non-commuting observables, Nature 538, 491 (2016).

[65] C. S. Jackson and C. M. Caves, How to Perform the Coherent Measurement of a Curved Phase Space by Continuous Isotropic Measurement. I. Spin and the Kraus-Operator Geometry of $\operatorname{SL}(2, \mathbb{C})(2021)$, ArXiv:2107.12396.

[66] K. Kraus and A. Böhm, States, Effects, and Operations: Fundamental Notions of Quantum Theory: Lectures in Mathematical Physics at the University of Texas at Austin Vol. 190 (Springer-Verlag, New York, Berlin, 1983).

[67] A. N. Jordan and M. Büttiker, Continuous Quantum Measurement with Independent Detector Cross Correlations, Phys. Rev. Lett. 95, 220401 (2005).

[68] E. Arthurs and J. L. Kelly, On the simultaneous measurement of a pair of conjugate observables, Bell Syst. Tech. J. 44, 725 (1965).

[69] M. A. Ochoa, W. Belzig, and A. Nitzan, Simultaneous weak measurement of non-commuting observables: A generalized Arthurs-Kelly protocol, Sci. Rep. 8, 15781 (2018).

[70] J. H. Shapiro, The quantum theory of optical communications, IEEE J. Sel. Top. Quantum Electron. 15, 1547 (2009). 
[71] C. M. Caves, J. Combes, Z. Jiang, and S. Pandey, Quantum limits on phase-preserving linear amplifiers, Phys. Rev. A 86, 063802 (2012).

[72] R. Ruskov, A. N. Korotkov, and K. Mølmer, Qubit State Monitoring by Measurement of Three Complementary Observables, Phys. Rev. Lett. 105, 100506 (2010).

[73] J. Atalaya, S. Hacohen-Gourgy, L. S. Martin, I. Siddiqi, and A. N. Korotkov, Multitime correlators in continuous measurement of qubit observables, Phys. Rev. A 97, 020104(R) (2018).

[74] J. Atalaya, S. Hacohen-Gourgy, L. S. Martin, I. Siddiqi, and A. N. Korotkov, Correlators in simultaneous measurement of non-commuting qubit observables, npj Quantum Inf. 4, 41 (2018).

[75] J. Atalaya, S. Hacohen-Gourgy, I. Siddiqi, and A. N. Korotkov, Correlators Exceeding One in Continuous Measurements of Superconducting Qubits, Phys. Rev. Lett. 122, 223603 (2019).

[76] J. Zhang and K. Mølmer, Prediction and retrodiction with continuously monitored Gaussian states, Phys. Rev. A 96, 062131 (2017).

[77] X.-B. Wang, T. Hiroshima, A. Tomita, and M. Hayashi, Quantum information with Gaussian states, Phys. Rep. 448, 1 (2007).

[78] A larger variance in, e.g., $\hat{X}$ indicates a greater uncertainty about the outcome of the $\hat{X}$ measurement. As a general principle, the more uncertainty there is about the outcome of a measurement, the more information one gains by performing that measurement. Thus we identify $g_{1}$ and $g_{2}$ as specifying the rate of an observer's information gain by making $\hat{X}$ and $\hat{P}$ measurements with strengths $\tau_{1}$ and $\tau_{2}$, respectively. For a related discussion, see Ref. [80].

[79] Note also that a straightforward application of the Heisenberg uncertainty principle $q_{3}+q_{5} \geq 1$ indicates that $\mathrm{g}_{1}+$ $g_{2} \geq\left(16+\tau_{1}+\tau_{2}\right)^{-1}$.

[80] K. C. Cylke, P. Lewalle, T. Noungneaw, H. M. Wiseman, A. N. Jordan, and A. Chantasri, Extremal Probability Paths for Diffusive Classical and Quantum Trajectories, In preparation (2022).

[81] Some system dynamics allow for multiple OP solutions connecting particular boundary conditions, in close analogy with the formation of caustics in ray solutions of optical problems. The uniqueness of the above solutions is a direct proof that this phenomenon cannot occur in the present case.

[82] L. D. Landau and E. M. Lifshitz, Mechanics, Third Edition: Volume 1 (Course of Theoretical Physics) (Butterworth-Heinemann, Oxford, 1976), 3rd ed.

[83] V. I. Arnold, Mathematical Methods of Classical Mechanics (Springer, New York, 1989).

[84] H. Goldstein, Classical Mechanics (Addison-Wesley, San Francisco, 1980).
[85] C. Elouard and A. N. Jordan, Efficient Quantum Measurement Engines, Phys. Rev. Lett. 120, 260601 (2018).

[86] A. N. Jordan, C. Elouard, and A. Auffèves, Quantum measurement engines and their relevance for quantum interpretations, Quantum Stud.: Math. Found. 7, 203 (2020).

[87] T. Sagawa and M. Ueda, Second Law of Thermodynamics with Discrete Quantum Feedback Control, Phys. Rev. Lett. 100, 080403 (2008).

[88] K. Jacobs, Second law of thermodynamics and quantum feedback control: Maxwell's demon with weak measurements, Phys. Rev. A 80, 012322 (2009).

[89] K. Jacobs, Quantum measurement and the first law of thermodynamics: The energy cost of measurement is the work value of the acquired information, Phys. Rev. E 86, 040106 (2012).

[90] E. Lutz and S. Ciliberto, Information: From Maxwell's demon to Landauer's eraser, Phys. Today 68, 30 (2015).

[91] N. P. Cottet, S. Jezouin, L. Bretheau, P. CampagneIbarcq, Q. Ficheux, J. Anders, A. Auffèves, R. Azouit, P. Rouchon, and B. Huard, Observing a quantum Maxwell demon at work, Proc. National Acad. Sci. 114, 7561 (2017).

[92] Y. Masuyama, K. Funo, Y. Murashita, A. Noguchi, S. Kono, Y. Tabuchi, R. Yamazaki, M. Ueda, and Y. Nakamura, Information-to-work conversion by Maxwell's demon in a superconducting circuit quantum electrodynamical system, Nat. Commun. 9, 1291 (2018).

[93] M. Naghiloo, J. J. Alonso, A. Romito, E. Lutz, and K. W. Murch, Information Gain and Loss for a Quantum Maxwell's Demon, Phys. Rev. Lett. 121, 030604 (2018).

[94] K. Jacobs, How to project qubits faster using quantum feedback, Phys. Rev. A 67, 030301 (2003).

[95] H. M. Wiseman and J. F. Ralph, Reconsidering Rapid Qubit Purification by Feedback, 8, 90 (2006).

[96] G. T. Landi, M. Paternostro, and A. Belenchia, Informational Steady-States and Conditional Entropy Production in Continuously Monitored Systems (2021), ArXiv:2103.06247.

[97] M. T. Mitchison, J. Goold, and J. Prior, Charging a Quantum Battery with Linear Feedback Control (2020), ArXiv:2012.00350.

[98] H. M. Chrzanowski, T. Symul, J. Bernu, B. Hage, A. Lund, T. C. Ralph, and P. K. Lam, in 2011 Conference on Lasers and Electro-Optics Europe and 12th European Quantum Electronics Conference (CLEO EUROPE/EQEC) (2011), p. 1.

[99] R. Simon, N. Mukunda, and B. Dutta, Quantum-noise matrix for multimode systems: $\mathrm{U}(n)$ invariance, squeezing, and normal forms, Phys. Rev. A 49, 1567 (1994).

[100] J. J. Sakurai and J. Napolitano, Modern Quantum Mechanics (Cambridge University Press, Cambridge, 2017), 2nd ed. 